\section{(1)}

CrossMark

\title{
European Respiratory Society guidelines for the diagnosis of primary ciliary dyskinesia
}

\author{
Jane S. Lucas ${ }^{1,2}$, Angelo Barbato ${ }^{3}$, Samuel A. Collins $\mathbb{1}^{1,2}$, Myrofora Goutaki $^{4,5}$ \\ Laura Behan ${ }^{1,2}$, Daan Caudri ${ }^{6,7}$, Sharon Dell ${ }^{8,9}$, Ernst Eber ${ }^{10}$, \\ Estelle Escudier ${ }^{11,12}$, Robert A. Hirst ${ }^{13}$, Claire Hogg ${ }^{14}$, Mark Jorissen ${ }^{15}$, \\ Philipp Latzin ${ }^{5}$, Marie Legendre ${ }^{11,12}$, Margaret W. Leigh ${ }^{16}$, Fabio Midulla ${ }^{17}$, \\ Kim G. Nielsen ${ }^{18}$, Heymut Omran ${ }^{19}$, Jean-Francois Papon ${ }^{20,21}$, Petr Pohunek ${ }^{22}$, \\ Beatrice Redfern ${ }^{23}$, David Rigau ${ }^{24}$, Bernhard Rindlisbacher ${ }^{25}$, \\ Francesca Santamaria ${ }^{26}$, Amelia Shoemark ${ }^{14}$, Deborah Snijders ${ }^{3}$, \\ Thomy Tonia ${ }^{4}$, Andrea Titieni ${ }^{19}$, Woolf T. Walker ${ }^{1,2}$, Claudius Werner ${ }^{19}$, \\ Andrew Bush ${ }^{14}$ and Claudia E. Kuehni ${ }^{4}$
}

@ERSpublications

International ERS guidelines recommend a combination of tests to diagnose primary ciliary dyskinesia http://ow.ly/sJhH304InBN

Cite this article as: Lucas JS, Barbato A, Collins SA, et al. European Respiratory Society guidelines for the diagnosis of primary ciliary dyskinesia. Eur Respir J 2017; 49: 1601090 [https://doi.org/10.1183/ 13993003.01090-2016].

ABSTRACT The diagnosis of primary ciliary dyskinesia is often confirmed with standard, albeit complex and expensive, tests. In many cases, however, the diagnosis remains difficult despite the array of sophisticated diagnostic tests. There is no "gold standard" reference test. Hence, a Task Force supported by the European Respiratory Society has developed this guideline to provide evidence-based recommendations on diagnostic testing, especially in light of new developments in such tests, and the need for robust diagnoses of patients who might enter randomised controlled trials of treatments. The guideline is based on pre-defined questions relevant for clinical care, a systematic review of the literature, and assessment of the evidence using the GRADE (Grading of Recommendations, Assessment, Development and Evaluation) approach. It focuses on clinical presentation, nasal nitric oxide, analysis of ciliary beat frequency and pattern by high-speed video-microscopy analysis, transmission electron microscopy, genotyping and immunofluorescence. It then used a modified Delphi survey to develop an algorithm for the use of diagnostic tests to definitively confirm and exclude the diagnosis of primary ciliary dyskinesia; and to provide advice when the diagnosis was not conclusive. Finally, this guideline proposes a set of quality criteria for future research on the validity of diagnostic methods for primary ciliary dyskinesia.

This article has supplementary material available from erj.ersjournals.com

Received: May 312016 | Accepted after revision: Aug 252016

These guidelines were endorsed by the ERS Science Council and Executive Committee in September 2016.

The guidelines published by the European Respiratory Society (ERS) incorporate data obtained from a comprehensive and systematic literature review of the most recent studies available at the time. Health professionals are encouraged to take the guidelines into account in their clinical practice. However, the recommendations issued by this guideline may not be appropriate for use in all situations. It is the individual responsibility of health professionals to consult other sources of relevant information, to make appropriate and accurate decisions in consideration of each patient's health condition and in consultation with that patient and the patient's caregiver where appropriate and/or necessary, and to verify rules and regulations applicable to drugs and devices at the time of prescription. 
Affiliations: ${ }^{1}$ Primary Ciliary Dyskinesia Centre, University Hospital Southampton NHS Foundation Trust, Southampton, UK. ${ }^{2}$ University of Southampton Faculty of Medicine, Academic Unit of Clinical and Experimental Medicine, Southampton, UK. ${ }^{3}$ Primary Ciliary Dyskinesia Centre, Dept of Woman and Child Health (SDB), University of Padova, Padova, Italy. ${ }^{4}$ Institute of Social and Preventive Medicine, University of Bern, Bern, Switzerland. ${ }^{5}$ Dept of Paediatrics, Inselspital, University Hospital of Bern, University of Bern, Bern, Switzerland. ${ }^{6}$ Telethon Kids Institute, The University of Western Australia, Subiaco, Australia. ${ }^{7}$ Dept of Pediatrics/Respiratory Medicine, Erasmus University, Rotterdam, The Netherlands. ${ }^{8}$ Division of Respiratory Medicine, The Hospital for Sick Children, Toronto, ON, Canada. ${ }^{9}$ Dept of Pediatrics and Institute of Health Policy Management and Evaluation, University of Toronto, Toronto, ON, Canada. ${ }^{10}$ Division of Paediatric Pulmonology and Allergology, Dept of Paediatrics and Adolescent Medicine, Medical University of Graz, Graz, Austria. ${ }^{11}$ Service de Génétique et Embryologie Médicales, Centre de Référence des Maladies Respiratoires Rares, Hôpital Armand Trousseau, Assistance Publique - Hôpitaux de Paris (AP-HP), Paris, France. ${ }^{12}$ Inserm UMR_S933, Sorbonne Universités (UPMC Univ Paris 06), Paris, France. ${ }^{13}$ Centre for PCD Diagnosis and Research, Dept of Infection, Immunity and Inflammation, University of Leicester, Leicester Royal Infirmary, Leicester, UK. ${ }^{14}$ Depts of Paediatrics and Paediatric Respiratory Medicine, Imperial College and Royal Brompton Hospital, London, UK. ${ }^{15}$ ENT Dept, University Hospitals Leuven, Leuven, Belgium. ${ }^{16}$ University of North Carolina at Chapel Hill, Chapel Hill, NC, USA. ${ }^{17}$ Paediatric Dept, Sapienza University of Rome, Rome, Italy. ${ }^{18}$ Danish PCD \& chILD Centre, CF Centre Copenhagen, Paediatric Pulmonary Service, Dept of Paediatrics and Adolescent Medicine, Copenhagen University Hospital, Rigshospitalet, Copenhagen, Denmark. ${ }^{19}$ Dept of Pediatrics, University Hospital Muenster, Münster Germany. ${ }^{20}$ AP-HP, Hôpital Kremlin-Bicetre, service d'ORL et de chirurgie cervico-faciale, Le Kremlin-Bicetre, France. ${ }^{21}$ Faculté de Médecine, Université Paris-Sud, Le Kremlin-Bicêtre, France. ${ }^{22}$ Paediatric Dept, Second Faculty of Medicine, Charles University and Motol University Hospital, Prague, Czech Republic. ${ }^{23}$ PCD Family Support Group. Milton Keynes, UK. ${ }^{24}$ Iberoamerican Cochrane Center, Barcelona, Spain. ${ }^{25}$ Kartagener Syndrom und Primäre Ciliäre Dyskinesie e. V., Steffisburg, Switzerland. ${ }^{26}$ Pediatric Pulmonology, Dept of Translational Medical Sciences, Federico II University, Azienda Ospedaliera Universitaria Federico II, Naples, Italy.

Correspondence: Jane S Lucas, Primary Ciliary Dyskinesia Centre, University Hospital Southampton NHS Foundation Trust, Southampton, S016 6YD, UK. E-mail: jlucas1dsoton.ac.uk

\section{Introduction}

Primary ciliary dyskinesia (PCD) represents a clinical and genetic heterogeneous group of respiratory ciliopathies, with reduced mucociliary clearance of the airways. The various mutations result in different clinical and pathological patterns, contributing to the challenges of diagnosis. There is no single gold standard diagnostic test for PCD [1]. Current diagnosis requires a combination of technically demanding investigations, including nasal nitric oxide (nNO), high-speed video microscopy analysis (HSVA) and transmission electron microscopy (TEM). Historically, clinicians used the saccharine test to screen for PCD, but this is no longer advocated [2]. Furthermore, more sophisticated diagnostic tests that might improve diagnostic accuracy (genotyping, immunofluorescence of ciliary proteins and electron microscopy tomography) are becoming increasingly available.

The availability of tests varies across Europe [3]. This has partially improved recently in response to collaborations including a former Task Force (2006-2009) of the European Respiratory Society (ERS) [2-4] and Framework Programme (FP)-7 funded BESTCILIA. The ERS Task Force published a consensus statement in 2009 [2] to guide diagnostic testing and BESTCILIA has recently introduced diagnostic testing into three countries where services did not previously exist. Since the 2009 statement, a number of groups and consortia have investigated the accuracy of various diagnostic tests for PCD, providing the opportunity to advance the state of diagnostics by developing evidence-based guidelines. Therefore, in 2014 a new PCD ERS Task Force consisting of adult and paediatric physicians from pulmonology and ear, nose and throat disciplines along with diagnostic scientists was established. It aimed to develop evidence-based guidelines for the diagnosis of PCD. This is important for the appropriate clinical management and prognosis of individual patients with suspected or eventually confirmed PCD to ensure patients with PCD are correctly

Support statement: J.S. Lucas, K.G. Nielsen, C.E Kuehni, C. Hogg, M.W Leigh and H. Omran received funding from the European Union Seventh Framework Programme (FP7/2007-2013) under grant agreement n8305404 (BESTCILIA). J.S. Lucas, L. Behan, W.T. Walker and S.A. Collins were supported by the NIHR Respiratory Biomedical Research Unit at the University Hospital Southampton NHS Foundation Trust (Southampton, UK) and AAIR Charity. A. Bush is an NIHR Senior Investigator and was supported by the NIHR Respiratory Disease Biomedical Research Unit at the Royal Brompton and Harefield NHS Foundation Trust and Imperial College London (both London, UK). M. Goutaki was supported by the following national grants: Bernese Lung League, Milena- Pro Kartagener foundation and Swiss National Foundation 32003B_162820/1. S. Dell and M.W. Leigh received funding from the NIH (U54HL096458) through the Genetic Disorders of Mucociliary Clearance Consortium, an initiative of the NIH Office of Rare Diseases Research at the National Center for Advancing Translational Science, and the National Heart, Lung and Blood Institute.

Conflict of interest: D. Rigau and T. Tonia are employees of the ERS. Other disclosures can be found alongside this article at erj.ersjournals.com 
diagnosed, whilst avoiding the problems of false-positive diagnoses. It should also ensure a definitive diagnosis before PCD patients are enrolled in randomised controlled clinical trials of treatment.

\section{Methods}

The methods are described in detail in the supplementary material.

\section{Task Force composition}

In brief, the panel consisted of a multidisciplinary group of clinicians and scientists with recognised expertise in the diagnosis of PCD; junior members/trainees affiliated to European PCD centres were active members of the committee (table S1). Methodologists from the ERS provided expertise in guideline development following the GRADE (Grading of Recommendations, Assessment, Development and Evaluation) approach for diagnostic tests [5]. Panel members disclosed potential conflicts of interest according to ERS policies at the start of the Task Force and prior to publication of this manuscript.

\section{Patient-important outcomes}

The GRADE approach emphasises the importance of recommendations based on the impact on patient-important outcomes [6]. The patient representatives to the Task Force fully endorsed that an accurate diagnosis was an important outcome, because it leads to a better recognition of their problems by physicians and more effective treatment, and thus improves their health and quality of life. This was confirmed by our questionnaire survey of $352 \mathrm{PCD}$ patients from 25 countries and 20 in-depth interviews [7]. However, diagnostic accuracy studies do not provide direct evidence for the improvement of patient-important outcomes; consequently, the confidence in results of test accuracy studies can be judged, at best, as moderate.

\section{Formulation of the topics and questions}

The Task Force members agreed that six facets of PCD diagnostics should be evaluated: clinical symptoms, nNO, HSVA, TEM, genotype and immunofluorescence labelling of ciliary proteins. We evaluated each test to see whether it should be included in the diagnostic pathway for PCD, using a "PICO" structured question: "Patients suspected of having PCD, Investigated by [nNO, TEM, etc.], when Comparing patients with a final positive or negative diagnostic outcome, what was the diagnostic accuracy (Outcome) of the test?" The PICO questions for each test were finalised during several rounds of teleconferences and email discussions (table S2).

The essential inclusion criterion for studies was that they must have included consecutive patients referred for PCD testing in whom the PCD diagnosis was then either confirmed or excluded. We excluded studies if patients had already had previous diagnostic testing. In the absence of such studies, in the narrative review we discussed case-control studies that compared PCD patients with healthy controls or patients suffering from other respiratory diseases (e.g. cystic fibrosis). Results from such studies cannot be generalised to the clinical situation, where patients with PCD must be distinguished from patients referred for similar complaints, but without PCD. Thus, the results from case-control studies are far less relevant for clinical care. The main limitation for this project was the lack of a gold standard diagnostic test for PCD. In the absence of this, we compared the diagnostic performance indicators (e.g. sensitivity and specificity) to the authors' final decision regarding positive/negative PCD diagnosis based on all available tests.

The Task Force also agreed on a list of less structured questions relevant to PCD diagnostics for the narrative discussion. As the evidence for these questions were not formally graded they were not used for recommendations.

\section{Literature search methods}

We searched Medline and Embase databases (accessed through Ovid) from January 1, 1996 to March 14, 2016. Full details are provided in the supplementary material. In brief, titles and abstracts were screened; the full text was then reviewed for papers that potentially fulfilled criteria for inclusion. These manuscripts were checked for completeness by the Task Force panel to ensure all data fulfilling the a priori inclusion criteria were present. PRISMA flow diagrams show the search process for each Working Group (figure S1).

\section{Quality of evidence and strength of recommendations}

We used the GRADE approach through the entire process, from grading the quality of evidence, to deciding on the strength of the recommendations [8,9]. Details are provided in the supplementary material, and include reasons for downgrading the confidence in the evidence (summary of evidence tables). Recommendations were made based on the strength of evidence and other factors, such as overall accuracy of the test (sensitivity and specificity), confidence in the net accuracy (range of sensitivity/ sensitivity from included studies and/or confidence intervals of net sensitivity and specificity), and considerations such as patient acceptability of the test, feasibility of testing and accessibility of the test. 
The four tests, where evidence-based recommendations were made, were all acceptable to the patient, feasible and acceptable.

\section{Consensus statement for confirming or excluding PCD}

We used a modified Delphi survey to reach a consensus regarding the use of diagnostic tests to definitively confirm and exclude the diagnosis of PCD, and to provide advice regarding patients who do not have a definitive diagnosis but diagnostic tests suggest that the diagnosis is highly likely or inconclusive. The methods are detailed in the supplementary material.

\section{Results}

The results of the evidence assessment gave rise to the recommendations listed in table 1 .

TABLE 1 Evidence-based recommendations for the use of each of the six tests considered for primary ciliary dyskinesia (PCD) diagnosis

Which patients should be referred for diagnostic testing?

Based on moderate confidence in the evidence:

1. We recommend that patients are tested for PCD if they have several of the following features: persistent wet cough; situs anomalies; congenital cardiac defects; persistent rhinitis; chronic middle ear disease with or without hearing loss; a history in term infants of neonatal upper and lower respiratory symptoms or neonatal intensive care admittance (strong recommendation)

2. Patients with normal situs presenting with other symptoms suggestive of PCD (as listed in recommendation 1) should be referred for diagnostic testing (strong recommendation)

3. Siblings of patients should be tested for PCD, particularly if they have symptoms suggestive of PCD (as listed in recommendation 1) (strong recommendation)

4. We recommend the use of combinations of distinct PCD symptoms and predictive tools (e.g. PICADAR) to identify patients for diagnostic testing (weak recommendation)

In patients suspected of having PCD should nasal nitric oxide be used as a diagnostic tool?

Based on moderate confidence in the evidence, we recommend that:

1. Nasal nitric oxide measurement should be used as part of the diagnostic work-up of school children aged $>6$ years and adults suspected of having PCD, preferably using a chemiluminescence analyser with a velum closure technique (strong recommendation)

2. In children aged $<6$ years suspected of having PCD, we suggest nasal nitric oxide measurement using tidal breathing as part of the diagnostic work-up (weak recommendation)

Remark: we suggest that patients presenting with a strong clinical history should undergo further testing, even if nasal nitric oxide is normal (weak recommendation)

In patients suspected of having PCD should HSVA be used as a diagnostic tool?

Based on low confidence in the evidence, we recommend:

1. HSVA, including ciliary beat frequency and beat pattern analysis, should be used as part of the diagnostic work-up of patients suspected of having PCD (weak recommendation)

2. Ciliary beat frequency should not be used without assessment of ciliary beat pattern in diagnosing PCD (strong recommendation)

3. To improve diagnostic accuracy of HSVA, CBF/P assessment should be repeated after ALI culture (strong recommendation)

In patients suspected of having PCD should TEM be used as a diagnostic tool?

Based on low confidence in the evidence, we recommend:

1. Ciliary ultrastructure analysis by TEM should be used as part of the diagnostic work-up of patients suspected of having PCD (strong recommendation)

2. Further diagnostic investigations should be performed in patients with normal ultrastructure if the clinical history is strong (strong recommendation) ${ }^{\#}$

3. In patients with hallmark ciliary ultrastructure defects for PCD further confirmatory diagnostic investigations are not required (strong recommendation) $)^{\text {? }}$

In patients suspected of having PCD, should genotyping be used as a diagnostic tool?

There were no studies that fulfilled inclusion criteria to answer this question. Statements to assist the clinician are made in the genetics sections but these are not evidence based. Therefore, we could not make formal recommendations as for other diagnostic procedures. However, we have provided a list of Task Force statements on genetics, which is based upon agreement between experts rather than published evidence.

In patients suspected of having PCD, should immunofluorescence be used as a diagnostic tool?

There were no studies that fulfilled inclusion criteria to answer this question. Statements to assist the clinician are made in the immunofluorescence sections but these are not evidence based. Therefore, we could not make formal recommendations as for other diagnostic procedures. However, we have provided a list of Task Force statements on immunofluorescence, based upon agreement between experts rather than published evidence.

TEM: transmission electron microscopy; HSVA: high-speed video microscopy analysis. \#: normal ciliary ultrastructure, as resolvable by TEM, does not exclude the diagnosis of PCD (16\% PCD-positive patients have TEM without a detectable defect); " : patients with hallmark ciliary ultrastructure defects for PCD labsence of outer dynein arms, combined absence of inner and outer dynein arms, inner dynein arm absence combined with microtubular disarrangement) assessed by TEM almost always have PCD (false-positive results are very rare $\approx 0.7 \%$ ). CBF/P: ciliary beat frequency/pattern; ALI: air-liquid interface. 


\section{Clinical features}

Summary of recommendations

Which patients should be referred for diagnostic testing?

Based on moderate confidence in the evidence:

1) We recommend that patients are tested for PCD if they have several of the following features: persistent wet cough; situs anomalies; congenital cardiac defects; persistent rhinitis; chronic middle ear disease with or without hearing loss; a history in term infants of neonatal upper and lower respiratory symptoms or neonatal intensive care admittance (strong recommendation).

2) Patients with normal situs presenting with other symptoms suggestive of PCD should be referred for diagnostic testing (strong recommendation).

3) Siblings of patients should be tested for PCD, particularly if they have symptoms suggestive of PCD (strong recommendation).

4) We recommend the use of combinations of distinct PCD symptoms and predictive tools (e.g. PICADAR) to identify patients for diagnostic testing (weak recommendation).

Review of evidence directly addressing the question "in patients suspected of having PCD, which clinical features predict a positive diagnosis"?

Our search identified 1269 studies of which two directly answered the question and were included in the quantitative synthesis (figure S1) and an additional six contributed to the narrative review. We excluded 1217 publications based on titles and abstracts. After full text review we excluded 44 of the remaining 52 studies because they did not fulfil the inclusion criteria (table S3).

Two studies, Behan et al. [10] and Shapiro et al. [11], were suitable to provide evidence for our recommendations. They included 1408 patients (table 2).

BEHAN et al. [10] analysed data from 868 consecutive paediatric and adult patients. 227 with inconclusive or incomplete results were excluded, leaving 641 for analysis. All patient data were collected through a proforma completed by a clinician prior to the diagnostic testing. They reported sensitivity and specificity of a large range of clinical features (table 2 and table S3). Wet cough did not discriminate well between PCD positive and negative patients (sensitivity 0.93 , specificity 0.15 ). Because it was the main reason for referral, it was present in virtually all patients. Neonatal chest symptoms and neonatal rhinitis had a high specificity (0.83 and 0.94 ), but a lower sensitivity $(0.27$ and 0.75$)$. The sensitivity and specificity for 25 clinical features are summarised in table 2 and described in detail in the supplementary material.

In addition to reporting on single symptoms, BEHAN et al. [10] developed a seven-point questionnaire-based prediction tool (PICADAR), to help predict the likelihood that a patient referred for evaluation of persistent wet cough has PCD. PICADAR was internally and externally (in a second cohort) validated and is the first clinical prediction tool developed for PCD. The score ranged from 0 to 14 . Sensitivity and specificity of a score of $>5$ were 0.90 and 0.75 , respectively, clearly better than single symptoms.

SHAPIRO et al. [11] analysed data from 767 consecutive paediatric and adult patients. Information on situs was determined by physicians at local consortium sites through review of radiology, surgery and cardiology reports and radiology images from participant medical records. Patients were divided into three situs categories: situs solitus, situs inversus and situs ambiguous (including heterotaxy).

Situs abnormalities were reported by BeHAn et al. [10] and SHAPIRO et al. [11] for a total of 1048 patients. The pooled sensitivity and specificity for the two papers (for any situs abnormality) was 0.508 and 0.939 , respectively (table S3).

\section{Narrative review of additional evidence}

LEIGH et al. [12] described a prospective cohort of 534 children with high suspicion of PCD, among whom many had a pre-existing diagnosis of PCD. Experts defined a priori and tested five clinical features apparent in early childhood and found four, either alone or in combination, to be predictive of PCD: 1) unexplained neonatal respiratory distress with supplemental oxygen requirement for $>24 \mathrm{~h}$ in term infants; 2) early-onset, year-round wet cough; 3) early-onset year-round nasal congestion; and 4) laterality defects [12]. Noll et al. [13] described a retrospective cohort of 323 patients with chronic cough referred for ciliary function analyses, and reported high specificity $(>0.9)$ for neonatal respiratory distress, persistent otitis media, situs inversus and bronchiectasis. CHIN et al. [14] retrospectively reviewed records of 118 patients referred for electron microscopy because of suspected PCD, and compared combinations of symptoms between patients with abnormal and normal electron microscopy, while excluding uncertain cases. They found more sino-nasal, middle ear and pulmonary symptoms in the abnormal group [11]. 


\begin{tabular}{|c|c|c|}
\hline Clinical manifestation & Sensitivity $(95 \% \mathrm{CI})$ & Specificity $(95 \% \mathrm{CI})$ \\
\hline \multicolumn{3}{|l|}{ Neonatal manifestations } \\
\hline Neonatal chest symptoms & $0.75(0.63-0.84)$ & $0.83(0.79-0.84)$ \\
\hline Neonatal rhinitis & $0.27(0.17-0.38)$ & $0.94(0.91-0.95)$ \\
\hline Neonatal respiratory support & $0.41(0.30-0.53)$ & $0.93(0.90-0.95)$ \\
\hline Neonatal unit admission & $0.61(0.49-0.72)$ & $0.86(0.83-0.89)$ \\
\hline \multicolumn{3}{|c|}{ Upper respiratory manifestations after the post-natal period } \\
\hline Chronic rhinitis & $0.81(0.70-0.89)$ & $0.43(0.38-0.47)$ \\
\hline Chronic serious otitis media & $0.57(0.45-0.69)$ & $0.81(0.77-0.84)$ \\
\hline Chronic acute otitis media & $0.33(0.23-0.45)$ & $0.75(0.71-0.79)$ \\
\hline Hearing loss & $0.49(0.38-0.61)$ & $0.84(0.81-0.87)$ \\
\hline Chronic ear perforation & $0.12(0.06-0.22)$ & $0.91(0.88-0.93)$ \\
\hline Ear surgery & $0.32(0.22-0.44)$ & $0.86(0.82-0.88)$ \\
\hline Chronic sinusitis & $0.28(0.19-0.40)$ & $0.76(0.72-0.79)$ \\
\hline \multicolumn{3}{|c|}{ Lower respiratory manifestations after the post-natal period } \\
\hline Chronic wet cough & $0.93(0.84-0.98)$ & $0.15(0.12-0.18)$ \\
\hline Recurrent wheeze & $0.48(0.36-0.60)$ & $0.62(0.57-0.65)$ \\
\hline Previous pneumonia & $0.41(0.30-0.53)$ & $0.65(0.61-0.69)$ \\
\hline Bronchiectasis & $0.29(0.20-0.41)$ & $0.68(0.64-0.72)$ \\
\hline \multicolumn{3}{|l|}{ Other manifestations (various ages) } \\
\hline Situs anomalies ${ }^{\#}$ & $0.51(0.46-0.56)$ & $0.94(0.92-0.95)$ \\
\hline Congenital heart disease & $0.08(0.03-0.17)$ & $0.98(0.97-0.99)$ \\
\hline Developmental delay & $0.11(0.05-0.20)$ & $0.94(0.91-0.96)$ \\
\hline Hydrocephalus & $0.01(0.00-0.08)$ & $0.99(0.98-1.00)$ \\
\hline Subfertility & $0.91(0.57-1.00)$ & $0.82(0.74-0.87)$ \\
\hline \multicolumn{3}{|l|}{ Family history (any age) } \\
\hline Primary ciliary dyskinesia in siblings & $0.24(0.15-0.35)$ & $0.98(0.97-0.99)$ \\
\hline Primary ciliary dyskinesia in extended family & $0.05(0.02-0.14)$ & $0.99(0.97-1.00)$ \\
\hline Asthma & $0.16(0.09-0.27)$ & $0.66(0.62-0.70)$ \\
\hline Bronchiectasis & $0.04(0.01-0.12)$ & $0.96(0.93-0.97)$ \\
\hline Otitis media & $0.07(0.02-0.16)$ & $0.89(0.86-0.92)$ \\
\hline \multicolumn{3}{|l|}{ Clinical scores } \\
\hline PICADAR score $>5$ & $0.90(0.81-0.96)$ & $0.75(0.70-0.80)$ \\
\hline
\end{tabular}

All data from Behan et al. [10] (641 eligible referrals, 75 (12\%) had primary ciliary dyskinesia). " : data on situs anomalies are from BeHAN et al. [10] and ShapIRo et al. [11] (767 referrals); ": data on subfertility are from a subgroup of 152 referrals where $11(7 \%)$ had primary ciliary dyskinesia.

BEUCHER et al. [15] compared patients with abnormal and normal electron microscopy in a retrospective cohort of 89 children suspected of PCD, excluding 21 uncertain cases and found that only situs inversus differed significantly between the groups. PIfFERI et al. [16] compared clinical symptoms in 98 patients with primary PCD versus secondary ciliary dyskinesia and statistically significant differences were found for situs inversus and severity of bronchiectasis. In the only publication that focused on neonates, Mullowney et al. [17] compared neonatal symptoms between 46 PCD patients and 46 controls with a history of neonatal respiratory distress syndrome, and found that lobar collapse, situs inversus and prolonged oxygen need were more common in infants with PCD. The combination of situs inversus, lobar collapse or oxygen need for $>2$ days had $87 \%$ (95\% CI 74-94) sensitivity and 96\% (85-99) specificity for PCD. A systematic review by GOUTAKI et al. [18] describes other case-control or case series studies on prevalence of clinical symptoms in PCD, which do not fulfil the inclusion criteria for this study. All studies are from developed countries, and it is probable that the predictive value of some symptoms would be different depending on geographical region; for example, sensitivity and specificity of bronchiectasis will be different in sub-Saharan Africa where bronchiectasis due to tuberculosis is common.

Key unanswered questions and research needs

Further research is needed using prospective cohort studies of patients referred with suspicion of PCD in whom clinical features are assessed in a standardised way before they are diagnosed. Analyses must be stratified by age. In particular, there is a need for prospective studies of neonates with neonatal respiratory distress syndrome. In addition, it might be helpful to combine information into clinical prediction scores, 
using state of the art approaches [17]. Validity of the different clinical features is also likely to vary depending on the population under evaluation. For instance, positive and negative predictive values of the symptoms strongly depend on the prevalence of the disease in the referral population and will be poorer in populations with a lower prevalence (i.e. in primary or secondary care compared to PCD referral centres). Sensitivity and specificity (shown in the table 2 ) do not depend on prevalence. Nevertheless, the mix in PCD phenotypes, and therefore the usefulness of different symptoms for prediction of PCD, is likely to differ between patients attending specialised clinics (e.g. ear nose and throat, pulmonology, and cardiology). For instance, while chronic cough will not distinguish between patients with and without PCD in a pulmonology clinic, chronic ear, nose and throat symptoms will not be distinctive in an ear, nose and throat clinic where (nearly) every patient has these complaints, and cardiac defects will not distinguish in a cardiology setting. Another factor to consider is that clinical features might differ between genetic variants (e.g. patients with CCNO variants show no situs anomalies but increased female infertility), so results vary with differences in prevalence of specific mutations in the evaluated population. Therefore, studies must be performed in specific healthcare settings and study populations, and consider age, sex and genetic abnormalities.

\section{Summary}

Relevant literature answering our question was extremely scarce (only two papers, of which one reported only on situs inversus), and results did not take severity of symptoms into account or stratify by age. Overall confidence in their results is moderate mainly because diagnostic performance does not inform downstream consequences of further clinical management based on the assessment of these symptoms.

Results suggest that clinical symptoms may help to distinguish between patients with and without PCD, but the positive predictive value (how many patients with a specific symptom have the disease) of single symptoms is low. Instead, the combination of suggestive symptoms might discriminate better, but this needs further study in different populations.

Wet cough starting in early childhood has been used as the initial selection criterion in most PCD studies. Therefore, it has a low discriminative value due to its high prevalence in both PCD positive and negative individuals recruited to these studies.

\section{Nasal nitric oxide}

Summary of recommendations

In patients suspected of having PCD, should nNO be used as a diagnostic tool?

Based on moderate confidence in the evidence, we recommend that:

1) $\mathrm{nNO}$ measurement should be used as part of the diagnostic work-up of schoolchildren aged $>6$ years and adults suspected of having PCD, preferably using a chemiluminescence analyser with a velum closure technique (strong recommendation).

2) In children aged $<6$ years suspected of having PCD, we suggest nNO measurement using tidal breathing as part of the diagnostic work-up (weak recommendation).

We suggest that patients presenting with a strong clinical history should undergo further testing, even if $\mathrm{nNO}$ is normal (weak recommendation).

\section{Explanation of the diagnostic test}

nNO is extremely low in PCD when compared to healthy and disease controls, for unknown reasons [19]. The accuracy of nNO as a diagnostic test in PCD varies by type of analyser, sampling method and age of patient [20].

Current guidelines recommend aspiration of gas from one nostril with gas entrained via the other naris to measure nNO using a stationary chemiluminescence analyser during a velum closure, such as breath hold or oral exhalation against resistance. The reading should be obtained from a technically acceptable plateau reading [21]. Whilst measurement during velum closure by chemiluminescence analyser is considered the "gold standard", this manoeuvre is not possible in all situations. In young children measurements during tidal breathing have been reported [22, 23]. Electromechanical portable analysers [23] are used where stationary chemiluminescence analysers are not available. There is currently no consensus over what threshold constitutes a positive or negative cut-off.

Although analysers report readings in parts per billion ( $\mathrm{ppb})$, this is influenced by the machine sampling rate, so the concentration is converted to $\mathrm{nL} \cdot \mathrm{min}^{-1}$ by the formula $\mathrm{nL} / \mathrm{min}=\mathrm{ppb} \times$ sampling rate in $\mathrm{L} \cdot \mathrm{min}^{-1}$. 
Review of evidence directly addressing the question "in patients suspected of having PCD, should nNO be used as a diagnostic tool?"

Our search identified 98 studies, of which 23 met inclusion criteria for qualitative assessment. Of these, four papers ( $n=588$ patients) assessed $\mathrm{nNO}$ in a cohort of patients suspected of $\mathrm{PCD}$ who eventually received either a positive or negative diagnosis, directly addressing the question (table 3 and table S4). The other 19 papers were excluded from informing the recommendations, but contributed to the narrative review (table S5).

MARTHIN et al. [24] measured nNO during breath hold, exhalation against resistance and tidal breathing. Sensitivity ranged from 0.92 (for breath hold) to 1.0 (oral exhalation against resistance) and specificity ranged from 0.80 (tidal breathing) to 0.96 (breath hold) [24]. LEIGH et al. [25] developed a threshold of $77 \mathrm{~nL} \cdot \mathrm{min}^{-1}$ using data from a PCD specialist centre and then trialled this cut-off in 155 consecutive patients at other sites. Comparing PCD-positive to indeterminate patients provided a sensitivity of 0.99 and specificity of 0.75 [25]. Lower specificity was because the diagnostic protocol only included electron microscopy and genetics, thus missing a number of true PCD cases ("indeterminate" rather than PCD negative). BEYDON et al. [22] reported a sensitivity of 0.91 and specificity of 0.86 for velum closure (cut-off $82 \mathrm{~nL} \cdot \mathrm{min}^{-1}$ ) and 0.90 and 0.97 for tidal breathing ( $40 \mathrm{~nL} \cdot \mathrm{min}^{-1}$; mean of five peaks). JACKSON et al. [26] used a cut-off of $30 \mathrm{~nL} \cdot \mathrm{min}^{-1}$ and reported sensitivity and specificity of 0.91 and 0.96 , respectively, to distinguish 34 PCD-positive from 267 PCD-negative patients. Further methodological details of these four studies are included in table S4.

Overall confidence in this evidence is moderate mainly because diagnostic performance is not informative of downstream consequences of further clinical management.

Narrative review of additional evidence

A number of studies that did not meet the inclusion criteria for making recommendations addressed important issues. Several studies used alternative methods for the measurement of $\mathrm{nNO}$ that do not use the American Thoracic Society/ERS guideline "gold standard" (velum closure, stationary analyser) [21]. Tidal breathing manoeuvres are useful, especially in those unable to perform a velum closure, but may be less discriminative. The study by MARTHIN et al. [23] of consecutive referrals found breath hold sensitivity of 0.92 and specificity of 0.96 compared to tidal breathing values of 0.93 and 0.80 (thresholds were breath hold $52.5 \mathrm{~nL} \cdot \mathrm{min}^{-1}$ and tidal breathing $-47.4 \mathrm{~nL} \cdot \mathrm{min}^{-1}$ ). However, BEYDON et al. [22] found increased accuracy of tidal breathing $\left(37.9 \mathrm{~nL} \cdot \mathrm{min}^{-1}\right.$ threshold; sensitivity 0.94 , specificity 0.92$)$ versus velum closure $\left(82.2 \mathrm{~nL} \cdot \mathrm{min}^{-1}\right.$, sensitivity 0.91 , specificity 0.86$)$. Measurement using portable analysers was assessed in two case-control studies. Using a portable analyser, MARTHIN et al. [23] compared PCD patients to those with cystic fibrosis and healthy controls. They found a sensitivity and specificity of 1.0 and 0.95 , respectively, for breath hold $\left(64 \mathrm{~nL} \cdot \mathrm{min}^{-1}\right.$ threshold) and 1.0 and 1.0 , respectively, for tidal breathing $\left(43 \mathrm{~nL} \cdot \mathrm{min}^{-1}\right)$ [23]. HARRIS et al. [27] studied 13 PCD and 37 disease control/healthy patients using tidal breathing and a portable analyser with a cut-off of $30 \mathrm{~nL} \cdot \mathrm{min}^{-1}$. Sensitivity and specificity were 1.0 and 0.95 , respectively [27].

Measurement of $\mathrm{nNO}$ in young children is possible; however, discrimination between PCD patients and controls is reduced as $\mathrm{nNO}$ is inversely proportional to age in healthy patients <12 years of age [28, 29]. One study showed that velum closure was possible in children as young as 3.9 years [20]. However, the majority of very young children are unable to co-operate with velum closure and so tidal breathing

TABLE 3 Summary of diagnostic accuracy of nasal nitric oxide from measurements in consecutive patients suspected of primary ciliary dyskinesia (PCD)

\section{First author [ref.] Study population}

\begin{tabular}{l}
\hline Marthin [24] \\
Leigh [25] \\
Beydon [22] 155 referrals (PCD: $n=71 ;$ \\
indeterminate: $n=84)$ \\
86 referrals: (PCD: $n=49 ;$ \\
non-PCD: $n=37)$
\end{tabular}

JACKSON [26]
301 referrals: (PCD: $n=34$; non-PCD: $n=267$ )

\section{Sampling method}

Threshold
$\mathrm{nL} \cdot \min ^{-1}$

Breath hold: $n=58$

Oral exhalation against resistance: $\mathrm{n}=37$

Tidal breathing: $n=97$

Oral exhalation, velum closure: $n=155$

Velum closure: $\mathrm{n}=74$

Tidal breathing (five peaks): $n=86$

Velum closure (breath hold or oral exhalation): $\mathrm{n}=301$

52.5
72.6
47.4
77
82.2

52.5

72.6

47.4

77

82.2

40

30

$0.90(0.74-0.98)$
$0.91(0.79-0.98)$

$0.90(0.78-0.97)$
Specificity (95\% CI)

$0.96(0.85-0.995)$

$0.94(0.79-0.99)$

$0.80(0.69-0.88)$

$0.75(0.64-0.84)$

$0.86(0.68-0.96)$

$0.97(0.86-0.999)$
$0.95(0.90-0.98)$ 
measurements have to be used. The studies by MARTHIN et al. [23] and BEYDON et al. [22] suggest that tidal breathing may produce similar accuracy to velum closure in adults; however, this has not been shown in children. The study of 117 consecutive referrals of all ages by MARTHIN et al. [23] found that the false-positive rate for children $<6$ years of age using tidal breathing was $39 \%$.

There is increasing evidence that some genetic mutations causing PCD may be associated with subtle beating abnormalities and nNO levels within the normal range. This includes two studies of PCD individuals with mutations encoding radial spoke head proteins, and one study in PCD individuals with abnormal nexin link composition due to GAS8 mutations associated with an nNO higher than usually seen in PCD [30-32]. This may partially explain the variability of the results and low diagnostic performance of nNO in some studies.

\section{Key unanswered questions and research needs}

Current evidence has shown that nNO is a useful test as part of the diagnostic process; however, there is no consensus on appropriate thresholds. Likewise, standardised protocols and thresholds need to be developed for tidal breathing, portable analysers and measurements, and normative data in younger children, particularly those $<6$ years of age. Further work on genotype-phenotype correlation can help in the interpretation of $\mathrm{nNO}$ levels in cases of diagnostic uncertainty in order to reduce the number of false-negative test results. There is no evidence that can lead us to recommend which patients with a normal nNO should be referred for further testing and this requires evaluation.

\section{Summary}

$\mathrm{nNO}$ is a highly accurate test for PCD when measured via a stationary chemiluminescence analyser using velum closure techniques with a sensitivity and specificity of $0.90-1.0$ and $0.75-0.97$, respectively. Tidal breathing technique or use of portable analysers are less sensitive and specific but may contribute to the diagnostic decision. Different studies have used different methods and cut-off values making it difficult to provide definite thresholds.

$\mathrm{nNO}$ is not sufficiently accurate to rule PCD in or out in isolation but considering that it is relatively easy to perform, is noninvasive and is affordable, the panel considered that it should be used as part of the diagnostic work-up of patients suspected of having PCD.

\section{High-speed video analysis}

Summary of recommendations

In patients suspected of having PCD, should HSVA be used as a diagnostic tool?

Based on low confidence in the evidence, we recommend:

1) HSVA, including ciliary beat frequency and beat pattern analysis, should be used as part of the diagnostic work-up of patients suspected of having PCD (weak recommendation).

2) Ciliary beat frequency should not be used without assessment of ciliary beat pattern in diagnosing PCD (strong recommendation).

To improve diagnostic accuracy of HSVA, CBF/p assessment should be repeated after air-liquid interface culture (strong recommendation).

\section{Explanation of the test}

PCD is related to abnormal ciliary function [33], which can be analysed ex vivo by assessment of ciliary activity in respiratory epithelium from the nose or bronchus. Ciliated cells can be observed immediately after sampling [34-36] and again after a period of culturing to differentiate PCD from secondary dyskinesia [37-40]. A video attached to a microscope records at high speeds (120-500 fps), and is replayed slower (30-60 fps) to review ciliary beat pattern and measure ciliary beat frequency [41]. Most studies have used analysis by expert microscopists, whilst several studies used computer analysis in an attempt to reduce subjectivity/observer bias. HSVA provides a permanent record that can be used for audit, expert advice or research.

\section{Review of evidence directly addressing the question "in patients suspected of having PCD, should} HSVA be used as a diagnostic tool?"

Our search identified 113 studies, of which 30 met inclusion criteria for qualitative assessment. (figure S1). Two studies (650 patients) assessed HSVA in cohorts suspected of PCD who eventually received either a positive or negative diagnosis, contributing to the evidence for recommendations (table 4). The other 28 papers did not meet the inclusion criteria for informing the recommendations, but contributed to our narrative review (table S6). 
TABLE 4 Summary of diagnostic accuracy of high-speed video analysis (HSVA) from evaluation in consecutive patients suspected of primary ciliary dyskinesia (PCD)

\begin{tabular}{|c|c|c|c|c|}
\hline First author [ref.] & $\begin{array}{l}\text { Study } \\
\text { population }\end{array}$ & Cilia assessment method & Sensitivity $(95 \% \mathrm{CI})$ & Specificity $(95 \% \mathrm{CI})$ \\
\hline PAPON [42] & $\begin{array}{c}25 \text { referrals } \\
\text { (10 PCD positive) }\end{array}$ & $\begin{array}{l}\text { HSVA beat frequency and quantitative } \\
\text { measurements of beat pattern }\end{array}$ & $0.96(0.89-0.98)$ & $0.95(0.91-0.98)$ \\
\hline JACKSON [26] & $\begin{array}{l}625 \text { referrals } \\
\text { (60 PCD positive) }\end{array}$ & HSVA beat frequency and subjective pattern & $1.0(0.94-1.00)$ & $0.93(0.91-0.95)$ \\
\hline
\end{tabular}

PAPON et al. [42] measured 12 parameters of ciliary beat pattern, including ciliary beat frequency. The distance travelled by the cilia tip weighted by the percentage of beating edges had the best sensitivity $(0.96)$ and specificity (0.95) to distinguish 10 PCD positive from 15 PCD negative patients [42]. Using this parameter in nine patients with previously inconclusive diagnoses, it was possible to support the diagnosis of PCD in four cases and exclude it in two. JACKSON et al. [26] found a sensitivity and specificity of 1.00 and 0.93 , respectively, for the combination of ciliary beat frequency measurement and beat pattern evaluation in a cohort of 625 referrals, including 60 PCD positive patients.

Overall confidence in this evidence is low, mainly because diagnostic performance is not informative of downstream consequences of further clinical management based on the assessment of HSVA and because of study limitations (HSVA was widely used as part of the reference standard and lack of blinding).

Narrative review of additional evidence

To date, there is no standardised method for cell processing and analysis. Respiratory epithelium can be collected using brush, curette or forceps, usually from the nose [43]. Ciliary function varies under differing conditions; for example, temperature and $\mathrm{pH}$, with some centres measuring at $37^{\circ} \mathrm{C}[26,40,42,44]$ and others at lower temperatures $[35,37,45]$. This will affect ciliary function and all centres need to define their own normative data until a consensus is reached to allow standardisation of methods and reporting between centres.

Equivocal results or abnormal results require repeat sampling or re-analysis following cell culture [38, 40, 46,47 ] since secondary defects are common. In a series of 712 patients, JoRISSEN et al. [47] assessed ciliary beat frequency and ciliary coordination in suspension culture (i.e. spheroids). 642 (20\%) non-PCD patients demonstrated abnormal ciliary activity before culture, but after culture $100 \%$ had normal ciliary activity. Conversely, in biopsies of 70 PCD patients (evaluable in 56), 20\% had a normal ciliary beat frequency and $10 \%$ had coordinated ciliary activity before culture. After culture, normal ciliary beat frequency was found in $7 \%$ of PCD patients but ciliary function was never normally coordinated, making this parameter more sensitive and specific than ciliary beat frequency measurement [47]. HiRsT et al. [40] reported ciliary beat frequency and pattern before and after air-liquid interface cultures in 158 patients. Before culture, most PCD and non-PCD patients exhibited a degree of functional ciliary abnormalities. After air-liquid interface culture, normal ciliary beat pattern was observed in all non-PCD patients, whilst in PCD patients ciliary beat pattern was uniformly abnormal. PIFFERI et al. [46] assessed the results of ciliary beat pattern and frequency analysis after suspension culture (i.e. spheroids) in nine subjects with inconclusive results on nasal brushings. After culture, four patients had abnormal ciliary beat pattern suggesting PCD diagnosis, two had secondary dyskinesia ciliary beat pattern and three remained inconclusive. Culture techniques are limited by success rates ranging from $54 \%$ to $83 \%[26,37,40]$.

Ciliary beat frequency measurement does not adequately differentiate PCD from non-PCD unless combined with ciliary beat pattern assessment. STANNARD et al. [48] found a sensitivity and specificity of 0.97 and 0.95 , respectively, for the percentage of dyskinetic epithelial edges whilst ciliary beat frequency alone only yielded a sensitivity and specificity of 0.87 and 0.77 , respectively. Moreover, ciliary beat frequency may be slow, normal or increased in PCD depending on the genotype [45].

Some ultrastructural defects and genetic mutations causing PCD may be associated with specific patterns of ciliary beating. In a cohort of 56 children, CHILvers et al. [44] reported virtually immotile cilia in patients with either a: combined inner and outer dynein arm (IDA and ODA) defect or an isolated ODA defect; stiff beat pattern in patients with either an isolated IDA or radial spoke with an IDA defect; and circular beating cilia in patients with a ciliary transposition defect. RAIDT et al. [45] studied ciliary beat pattern according to the genetic variants of PCD. Although numbers associated with some genes were extremely small, the data supports the linking of a PCD causing gene with particular ciliary beat patterns: cilia from patients with mutations in ODA causing genes (DNAH5, DNAI1, DNAI2, ARMC4) showed minimal residual movements. In patients with DNAH11 mutations (normal ultrastructure), cilia exhibited 
a hyperkinetic ciliary beat pattern with reduced proximal axonemal bending. Some genetic defects, such as GAS8 mutations, can result in subtle defects that are hardly detectable by HSVA [30].

Key unanswered questions and research needs

Current evidence suggests that HSVA is a useful test as part of the diagnostic process, but there is no consensus on appropriate cell processing and method of ciliary assessment. Likewise, standardised protocols and thresholds need to be developed for ex vivo analysis of ciliary beat pattern. Further work on genotype/ultrastructural phenotype correlation can help in interpretation of HSVA parameters in cases of diagnostic uncertainty.

\section{Summary}

HSVA is an accurate test for PCD when performed by experienced observers combining ciliary beat frequency measurement and pattern analysis (sensitivity and specificity of $0.95-1.00$ and $0.93-0.95$, respectively). Culturing the respiratory cells may contribute to improve the accuracy of HSVA, in particular to rule out false positives.

HSVA is not sufficiently standardised to rule PCD in isolation in or out. Considering that optimal conditions in functional evaluation of cilia remains to be defined, the panel considered that HSVA should be performed by experienced staff as part of the diagnostic work-up of patients suspected of having PCD. This might impair the availability of the test in many centres.

\section{Transmission electron microscopy}

Summary of recommendations

In patients suspected of having PCD, should TEM be used as a diagnostic tool? Based on low confidence in the evidence, we recommend:

1) Ciliary ultrastructure analysis by transmission electron microscopy should be used as part of the diagnostic work-up of patients suspected of having PCD (strong recommendation).

2) Further diagnostic investigations should be performed in patients with normal ultrastructure if the clinical history is strong (strong recommendation).

3) In patients with hallmark ciliary ultrastructure defects for PCD further confirmatory diagnostic investigations are not required (strong recommendation).

Explanation of the diagnostic test

In 1976, Afzelius [33] demonstrated that TEM could be used to detect ultrastructural defects of cilia in patients with primary ciliary dyskinesia. Subsequently, for many years TEM was considered the "gold standard" diagnostic test for PCD. However, several genetic studies have demonstrated that an increasing number of distinct genetic PCD sub-types (e.g. due to DNAH11 mutations) cannot be diagnosed by TEM [49]. Thus, TEM cannot rule out PCD diagnosis.

Respiratory epithelium is usually sampled from the inferior turbinate of the nose by brush or curette biopsy or from the lower respiratory tract during bronchoscopy. The epithelium is chemically fixed with glutaraldehyde, processed and embedded into blocks, which are sectioned with an ultramicrotome. Staining with heavy metals (lead and uranyl) provides contrast. Assessment of cilia from healthy cells in transverse sections is made using TEM [50,51]. The number of cilia and cells analysed varies between centres; unless sufficient numbers are assessed, defects caused by mutations in genes that cause intermittent defects are likely to be missed. Considerable expertise is required to perform TEM and interpret results; expenditure for equipment and running costs are high.

The normal ultrastructural ciliary arrangement in a transverse section is a circle of nine microtubule doublets, each with a pair of dynein arms, plus a central pair of microtubules (figure 1). There are a number of ultrastructural phenotypes associated with a diagnosis of PCD (figure 2). The majority of cases are due to a lack of dynein arms; other defects include disorganisation of the microtubular doublets or loss of the central microtubular pair (table 5). Some patients with PCD have apparently normal ciliary ultrastructure, as seen by TEM. Secondary ciliary dyskinesia can be associated with transient ultrastructural abnormalities, such as compound cilia, axonemal blebs or additional tubules, which must not be confused with PCD.

Review of evidence directly addressing the question "in patients suspected of having PCD, should TEM be used as a diagnostic tool?"

We identified and screened 370 studies, of which the full texts of 46 were assessed for eligibility. Of the 17 that met inclusion criteria for qualitative assessment (table S7), 11 papers (3200 patients) assessed TEM in 


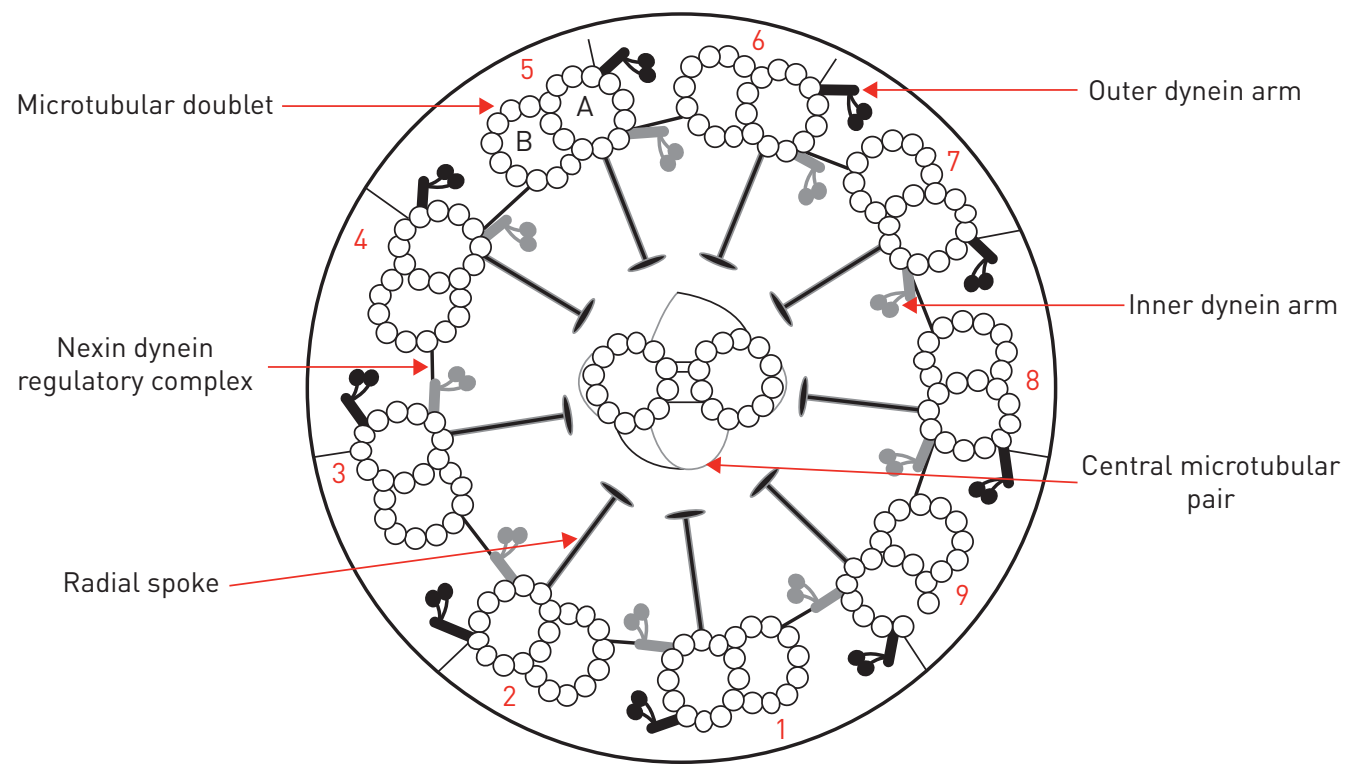

FIGURE 1 Diagram of normal ultrastructure of the ciliary axoneme in transverse section.

a cohort of patients suspected of PCD who eventually received either a positive or negative diagnosis, contributing to the evidence for recommendations (figure S1).

Tables 5 and 6 summarise the 11 studies addressing the question. The sensitivity calculated from each study ranged between 0.71 and 1.00 and the specificity between 0.92 and 1.00 . There were five false-positive patients in two studies. PAPON et al. [42] identified two false-positive results; one was a child with severe asthma without recurrent upper airway infection who had short or absent ODA concerning 44\% of nasal cilia and $90 \%$ of bronchial cilia, the other an adult with situs inversus and nasal polyposis without lower airway symptoms who had absent IDA without microtubular disorganisation (personal communication). Munkholm et al. [57] identified three false positives in individuals each eventually thought to have secondary ciliary dyskinesia, two of whose clinical phenotype improved becoming asymptomatic and a third who had severe asthma. Total specificity, when combining all 11 studies, was $>0.99$.

The quality of evidence was rated low because diagnostic performance is not informative of downstream consequences of further clinical management and because of study limitations (frequent use of TEM in the reference standard and lack of blinding). However, the very low rate of false positives and the very high specificity of TEM led to strong recommendations.

\section{Narrative review of additional evidence}

Assessment of the proportion of TEM defects in patients with PCD was made following a review of all manuscripts (post 1996) describing a cohort of more than 50 individuals $[26,42,48,56,58,59]$ (table 4). ODA defects (26-59\%) and combined ODA and IDA defects (6-39\%) were the most commonly observed. The recommendations below refer to common hallmark defects (absence of ODA, combined absence of IDA and ODA, IDA absence combined with microtubular disarrangement). Isolated IDA defects by TEM are controversial. Several studies acknowledge that IDA are difficult to visualise by TEM [42, 60, 61] and repeat analysis has been recommended before confirming a diagnosis [62]. For central pair defects the ciliary defect is usually present in a minority of cilia making the diagnosis difficult especially since patients do not have situs inversus.

Evidence for add on techniques to improve electron microscopy in the diagnosis of PCD was reviewed. Computer-assisted analysis has been reported to enhance the visualisation of dynein arms and consequently improve the sensitivity of electron microscopy $[60,61]$. Electron tomography is an advanced TEM technique allowing visualisation of structures in three-dimension. A series of TEM images are acquired by tilting the specimen stage at regular increments around two perpendicular axes. Images from both tilt series are then aligned into a single three-dimensional high-resolution projection. If a structural feature is repeated within a tomogram, it can be enhanced through sub-tomographic averaging; a technique in which software extracts the chosen common features and makes comparison by cross correlation. Electron tomography has been shown to improve three-dimensional visualisation and resolution of cilia allowing identification of patients with HYDIN and DNAH11 gene defects in a research setting [63]. The use of tomography for diagnosis has not been evaluated. 

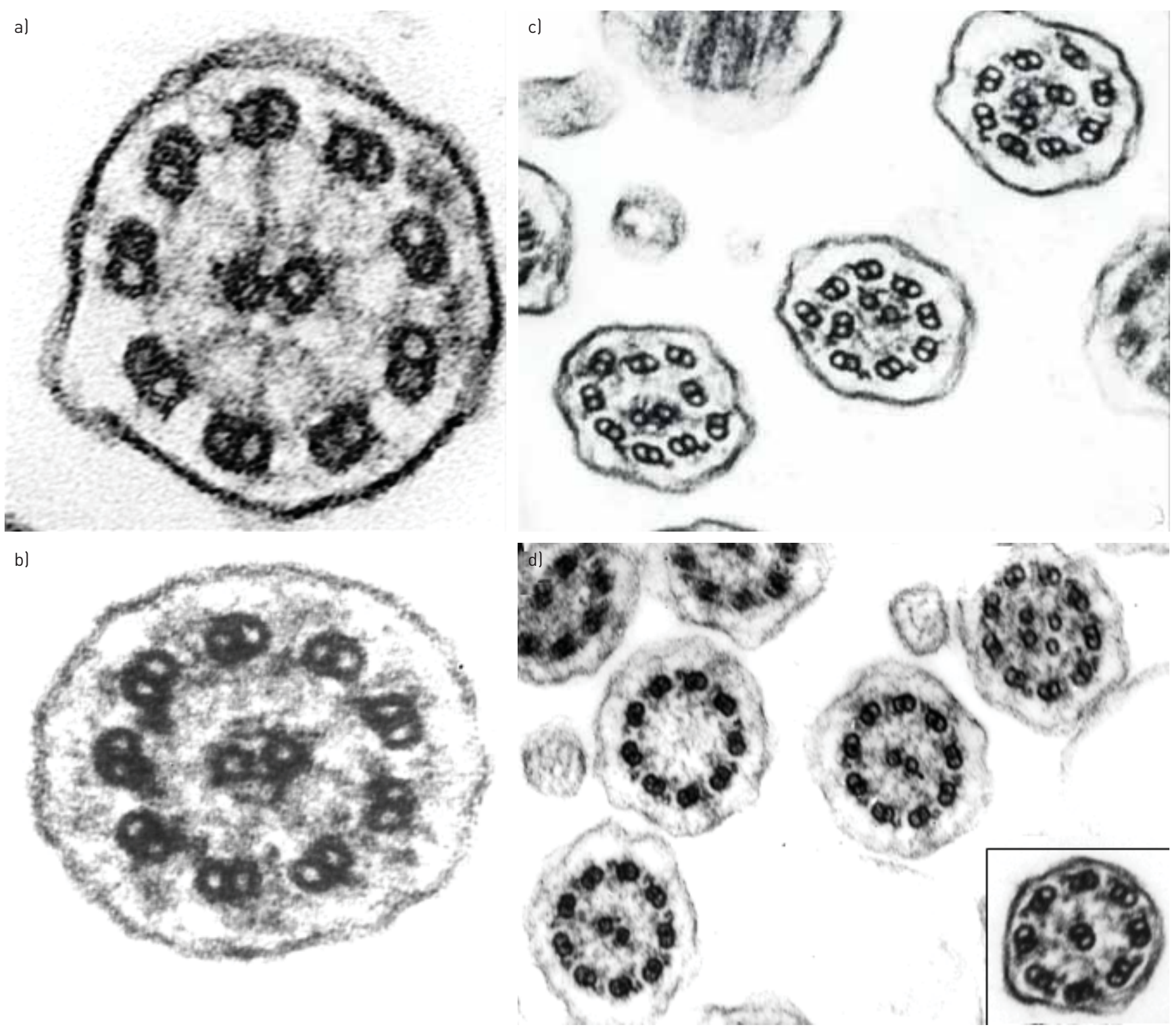

FIGURE 2 Electron microscopy images of primary ciliary dyskinesia defects. al Inner and outer dynein arm defect, b) outer dynein arm defect, cl inner dynein arm and microtubular disarrangement and d) central pair and transposition defect.

Our evidence review considered only conclusive results. Reported rates of inconclusive results ranged from $1.7 \%$ to $28.6 \%[26,37,39,58]$. This was attributed to poor sampling technique or the presence of secondary changes to the cilia. Seven of the 11 studies reported measures to avoid sampling during or immediately after an upper respiratory tract infection to improve adequacy and minimise secondary ciliary ultrastructural change.

Cell culture techniques that induce ciliogenesis from human biopsies are used in a number of PCD diagnostic centres. Two techniques to induce basal cell proliferation and ciliated cell differentiation have been described for PCD diagnosis [37, 40]. Jorissen et al. [37] established a submerged culture technique and the air-liquid interface technique was first described for PCD diagnosis by HiRsT et al. [40]. Both methods have shown that the TEM axoneme structure is conserved after cell culture in normal and PCD subjects, and they have been shown to reduce secondary damage $[37,40]$. TEM following culture has the potential to aid diagnosis of reduced generation of multiple motile cilia [64].

Key unanswered questions and research needs

Basic scientific research must improve the technique of TEM and identify PCD in those with "normal ultrastructure". The diagnostic community requires standardised protocols and consensus on terminology, 
TABLE 5 Characteristics of the ultrastructural defects described in nine studies directly addressing the PICO question using transmission electron microscopy to diagnose primary ciliary dyskinesia

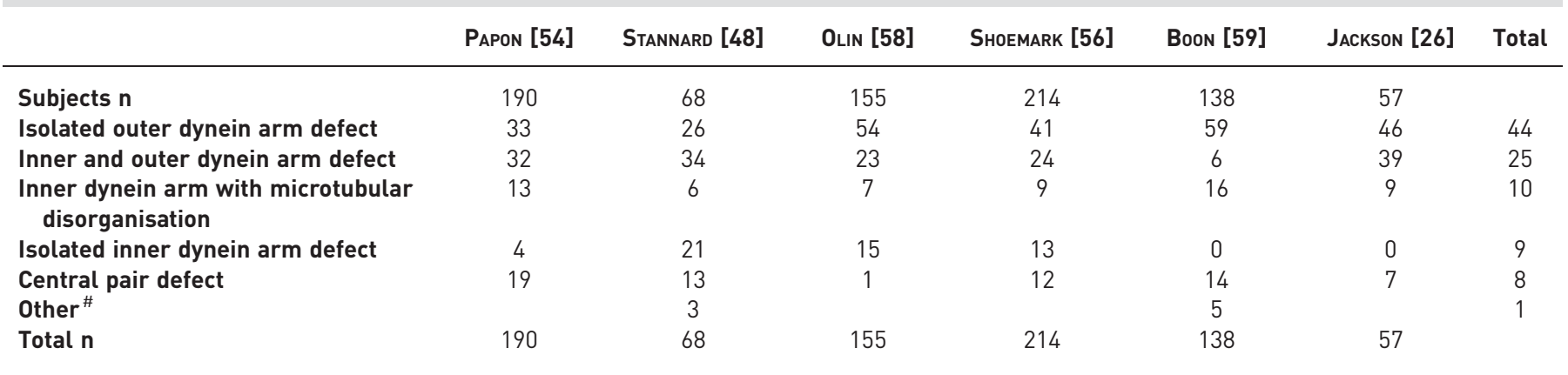

Data are presented as percentage, unless otherwise stated. ${ }^{\#}$ : include ciliary aplasia, disorientation and extra microtubules.

especially regarding the number and proportion of cilia required to make a diagnosis. True relevance and prevalence of IDA and other rare defects needs to be confirmed.

\section{Summary}

TEM is a highly specific test to confirm a diagnosis of PCD and is a key part of the diagnostic work. However, some patients with PCD have apparently normal ultrastructure and therefore TEM should not be used in isolation to exclude a diagnosis.

All 11 studies were retrospective analyses of cohorts of patients with clinical suspicion of PCD, the largest of which spanned time-periods of 20 years $[54,56]$. Further downgrading was due to the use of TEM as the reference standard and lack of blinding, resulting in grading of evidence as low.

\section{Genetics}

In patients suspected of having PCD, should genotyping be used as a diagnostic tool? There were no studies that fulfilled the inclusion criteria to answer this question.

\section{Explanation of the diagnostic test}

PCD is a genetically heterogeneous disorder. As with autosomal recessive disorders in general, disease is more likely in offspring from consanguineous relationships, and has a 1:4 probability from any conception where both parents are healthy carriers. To date, mutations in more than 30 genes have been reported to cause PCD (table 7). A more detailed explanation of the PCD-associated genes is presented in the supplementary material.

To establish the genetic diagnosis, non-ambiguous bi-allelic mutations in autosomal recessive PCD and hemizygous mutations in X-linked PCD should be identified. The majority of reported mutations are nonsense, frameshift or splice mutations while missense mutations are identified in a minority of cases. Most of the mutations are private, but founder mutations (e.g. in DNAI1 [105] and DNAH5 [95]) and

TABLE 6 Sensitivity and specificity of the 11 studies directly addressing the PICO question using transmission electron microscopy to diagnose primary ciliary dyskinesia

\begin{tabular}{lcccc} 
First author [ref.] & Study population $\mathbf{n}$ & Conclusive diagnostic result reached $\mathbf{n}$ & Sensitivity (95\% CI) & Specificity (95\% CI) \\
\hline JoRISSEN [37] & 812 & 468 & $0.71(0.61-0.81)$ & $1.0(0.99-1.0)$ \\
PIFFERI [52] & 64 & 62 & $0.75(0.48-0.93)$ & $1.0(0.93-1.0)$ \\
PIFFERI [39] & 59 & 56 & $0.77(0.50-0.93)$ & $1.0(0.88-1.0)$ \\
HIRST [53] & 231 & 187 & $0.82(0.77-0.86)$ & $1.0(0.98-1.0)$ \\
PAPON [54] & 1149 & 793 & $0.92(0.62-1.0)$ & $1.0(0.99-1.0)$ \\
OLM [55] & 24 & 24 & $0.83(0.52-0.98)$ & $1.0(0.79-1.0)$ \\
PAPON [42] & 34 & 28 & $0.88(0.83-0.91)$ & $1.0(1.0-1.0)$ \\
SHOEMARK [56] & 1182 & 1031 & $0.96(0.87-1.0)$ & $1.0(0.95-1.0)$ \\
HIRST [40] & 165 & 122 & $0.83(0.61-0.95)$ & $0.92(0.79-0.98)$ \\
MUNKHOLM [57] & 239 & 61 & $0.79(0.68-0.88)$ & $1.0(0.99-1.0)$
\end{tabular}


TABLE 7 Overview of primary ciliary dyskinesia causing genes and their associated findings by transmission electron microscopy (TEM) and immunofluorescence analyses

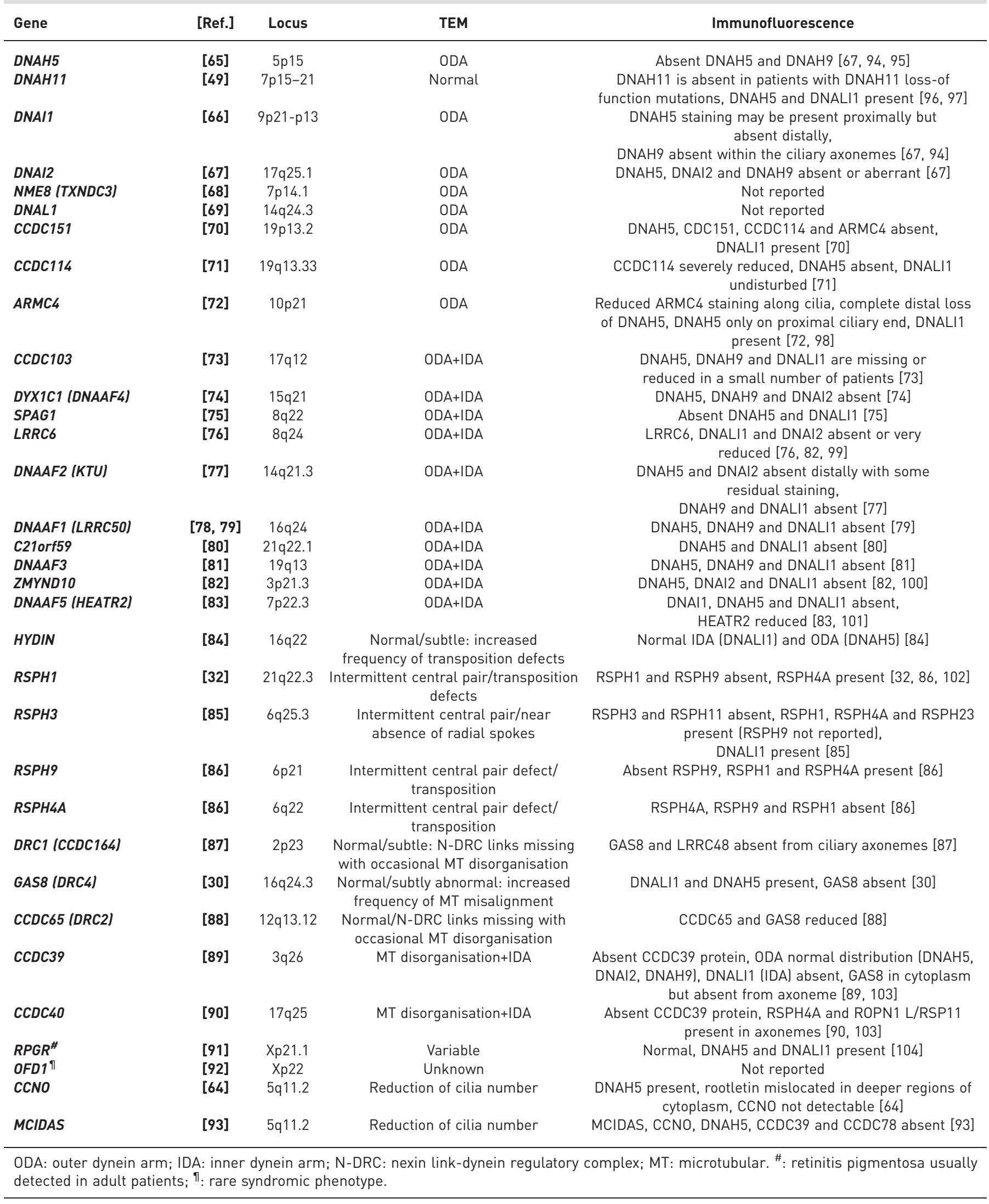


mutational hot spots (e.g. CCNO [106]) have been reported. The ranking of the effect of the mutations should follow international recommendations [107]: benign (class 1), likely benign (class 2), unknown significance (class 3), likely pathogenic (class 4), pathogenic (class 5).

The associations between genotype and structural defects documented by TEM and/or immunofluorescence are well established, but much less is known about gene HSVA associations. With studies based on small numbers of patients and often a limited number of videos per patient [45], there is insufficient data to correlate mutations within a gene with dyskinesia phenotype. To date, our knowledge suggests that disease-causing mutations in DNAH5 are always associated with predominantly static cilia whilst mutations in different regions of DNAH11 can lead to either static cilia or hyper frequent, stiff cilia [45, 108]. Therefore, to confirm the genetic cause of PCD, the ultrastructural defect and gene should correlate; in the future, we may be able to use gene mutation ciliary pattern correlations as further support.

In principle, all DNA sequencing technologies can be applied for genetic testing in patients with a confirmed PCD or a high suspicion of PCD (see supplementary material). However, due to the high number and the huge size of PCD genes high-throughput techniques are now widely used. The yield of allele-specific approaches is low in PCD given the high genetic and allelic heterogeneity. Detected mutations should be confirmed by Sanger sequencing and checked for segregation in the parents. Genetic laboratories have to be aware that large heterozygous genomic deletions have been reported in PCD individuals that might be missed by DNA sequencing technologies. Homozygous and heterozygous intragenic large duplications and deep intronic mutations are also missed by sequencing techniques. The detection of intragenic deletions and duplications will benefit from the fine set-up of targeted next generation sequencing panels; however, this approach requires specific development and sensitivity assessment. All techniques, especially the second line approaches, benefit from the knowledge of the ultrastructural defect of the patient in order to assess the relevance of the molecular findings. The $\approx 30$ PCD genes implicated to date encompass $>700$ exons and thus it is not unusual to identify a heterozygous variant in a gene that is obviously not responsible for the disease of the patient based on ultrastructural data. Cell and whole organism models can be used to confirm that a gene is disease causing.

Review of evidence directly addressing the question "in patients suspected of having PCD, should genotyping be used as a diagnostic tool?"

Searches identified 462 studies, of which 95 met inclusion criteria for qualitative assessment (table S8). Most studies included patients with confirmed PCD with the aim of identifying novel genes rather than diagnostic cohorts. There were no studies that fulfilled the inclusion criteria for quantitative assessment.

\section{Narrative review of additional evidence}

In populations with confirmed or highly suspected PCD diagnosis, it is possible to identify genetic causation in $50-75 \%$ of cases $[109,110]$. The sensitivity of genetic testing as a first-line diagnostic test for PCD is currently unknown but is likely to be low. With the identification of further PCD genes and high-throughput sequencing technologies, PCD genetic testing as "stand alone" testing might be considered in the future. Genotyping is useful in instances where confirmation of the diagnosis is difficult by other approaches (e.g. DNAH11, CCNO, MCIDAS and RSPH genes mutations). The detection of bi-allelic disease-causing mutations in autosomal recessive PCD or hemizygous mutations in X-linked PCD is highly specific.

Most studies to identify novel PCD gene defects used ultrastructural defects detected by routine TEM as the starting point for the genetics search; therefore, the likelihood to identify mutations in PCD with ultrastructural defects is higher than in PCD devoid of ultrastructural defects. This underscores the need not to rely on TEM as the sole diagnostic test for PCD.

Reports of mutations in specific genes typically relate to small numbers of patients and are not necessarily ethnically representative; therefore, the contribution of each gene should be interpreted with caution. Studies testing for DNAH5 and DNAI1 mutation suggest that these mutations account for $~ 50-70 \%$ of cases of ODA defects $[94,95,111,112]$. Mutations in CCDC39 or CCDC40 [89, 90, 103, 113] account for almost all PCD individuals with microtubular disorganisation and absence of IDA. Of 58 unrelated PCD patients with normal ultrastructure, $22 \%$ had bi-allelic mutations in DNAH11 [96]. Mutations in the genes encoding radial spoke head and stalk proteins (RSPH1, RSPH3, RSPH4A, RSPH9), HYDIN and nDRC proteins (DRC1, CCDC65, GAS8) can cause PCD with normal or subtly abnormal ultrastructure (table 7); to date, the contribution of these genes to the prevalence of PCD has not been determined.

A systematic population-based genetic Israeli study revealed that RGMC may be more frequent (6\%) in their particular PCD populations than previously estimated [106].

Genetic analyses have shown that mutations in the RGMC genes CCNO and MCIDAS, as well as the genes encoding radial spoke proteins (RSPH1, RSPH3, RSPH4A, RSPH9) and the central pair associated protein 
HYDIN, do not result in laterality defects. In addition, to date all PCD individuals carrying bi-allelic mutations in genes encoding nDRC proteins such as CCDC164, CCDC65 and GAS8 did not exhibit any situs abnormalities.

Given the large size of the regions sequenced in PCD patients, it is not unusual to identify one or several rare missense variants that are not linked to the disease. Great care should be taken to interpret those variants. It is important to perform segregation analysis for those variants and their interpretation can rely on expert labs. In some cases immunofluorescence microscopy has been proven to be a useful tool to determine pathogenicity of missense mutations in PCD individuals with mutations in RSPH4A and RSPH9 that encode radial spoke head proteins [86]. However, immunofluorescence analysis can be normal if the mutated protein is still expressed and correctly assembled within the axonemes, such as reported for DNAH11 missense mutations [97].

We did not find evidence to either confirm or refute genotyping as a diagnostic test for PCD. Whilst there is a need for evidence of the utility of genotyping in a diagnostic setting, table 8 summarises the Task Force assessment of currently published evidence in genetic testing in PCD.

\section{Key unanswered questions and research needs}

The role of genetic testing is not well defined in the PCD diagnostic pathway. We need studies to investigate the accuracy and limitations of genetics as a diagnostic tool for PCD. The standards for diagnostic testing for PCD need defining.

\section{Summary}

We were unable to determine the accuracy of genetic testing due to lack of suitable studies. Several studies have identified the genes responsible in patients with confirmed PCD, suggesting that genetic testing identifies the gene in $\sim 65 \%$ of cases; this is likely to increase as more genes are identified. The question of diagnostic accuracy should be revisited as new data become available.

\section{Immunofluorescence}

In patients suspected of having PCD, should immunofluorescence be used as a diagnostic tool? There were no studies that fulfilled inclusion criteria to answer this question.

\section{Explanation of the diagnostic test}

Labelling of ciliary proteins was developed to improve understanding of the impact of disease-causing genes on ciliary proteins [116]. Specific antibodies with secondary fluorescent tags localise to proteins in human respiratory epithelial cells and are visualised by fluorescent or confocal microscopy. A number of antibodies against ciliary proteins are available, including antibodies targeting the ODA, IDA, radial spoke head and dynein regulatory complex proteins. An example of this technique is shown in figure 3.

Respiratory epithelial cells in suspension are placed onto glass slides, air dried and fixed. The cells are incubated with antibodies to ciliary protein not implicated in PCD (e.g. acetylated tubulin) to label the axoneme and combined with antibodies of interest produced in a different species (e.g. anti-DNAH5 to identify ODA structures).

\section{TABLE 8 Summary of the Task Force consensus on the published evidence on genetic testing in primary ciliary dyskinesia} (PCD) diagnostics

Whilst further evidence in a diagnostic setting is required, experts of the Task Force agreed:

1. Genetic testing to confirm diagnosis can be performed in PCD individuals diagnosed by other means (e.g. HSVA, TEM, IF) or in individuals with high clinical suspicion for PCD (typical clinical findings, low nNO) and no availability of other investigations, such as HSVA, TEM or IF. A negative genetic test does not exclude PCD.

2. Genetic testing can also be performed to establish diagnosis in patients highly suspected of PCD and in whom HSVA, TEM or IF failed to confirm the diagnosis, as can be the case for patients with DNAH11, CCNO, MCIDAS or RSPH gene mutations.

3. Genetic testing and interpretation of results should follow national and international best practice guidelines [114, 115].

4. Genetic diagnosis has to be consistent with the clinical and TEM/IF/HSVA phenotype, or diagnosis reconsidered if the picture is inconsistent.

5. Allelic segregation analysis within the family (especially in both parents) is important to confirm the genotype in the probands (to differentiate between homozygosity and hemizygosity, and between compound heterozygosity and a complex allele).

6. Genetic testing in probands and in their relatives is helpful for genetic counselling to inform reproductive choices.

7. In the future genetic testing might be important for genotype specific therapy.

HSVA: high-speed video microscopy analysis; TEM: transmission electron microscopy; IF: immunofluorescence; nNO: nasal nitric oxide. 
a)
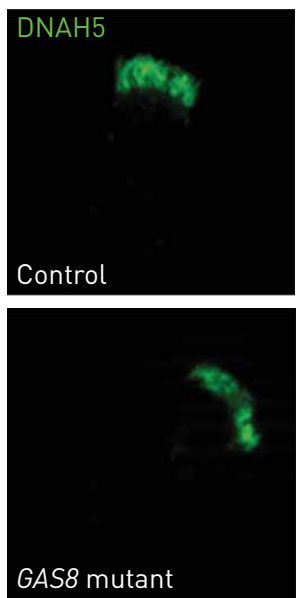

b)
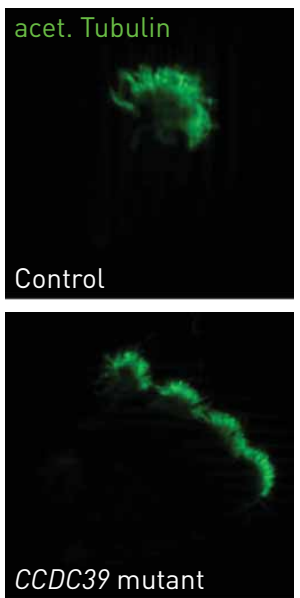

c)
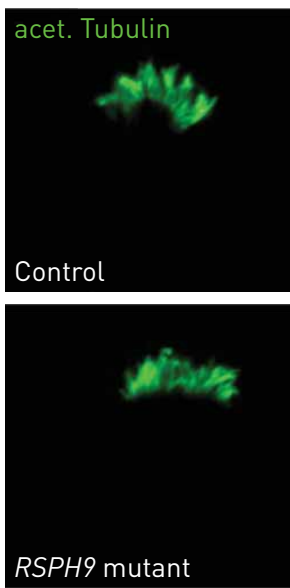
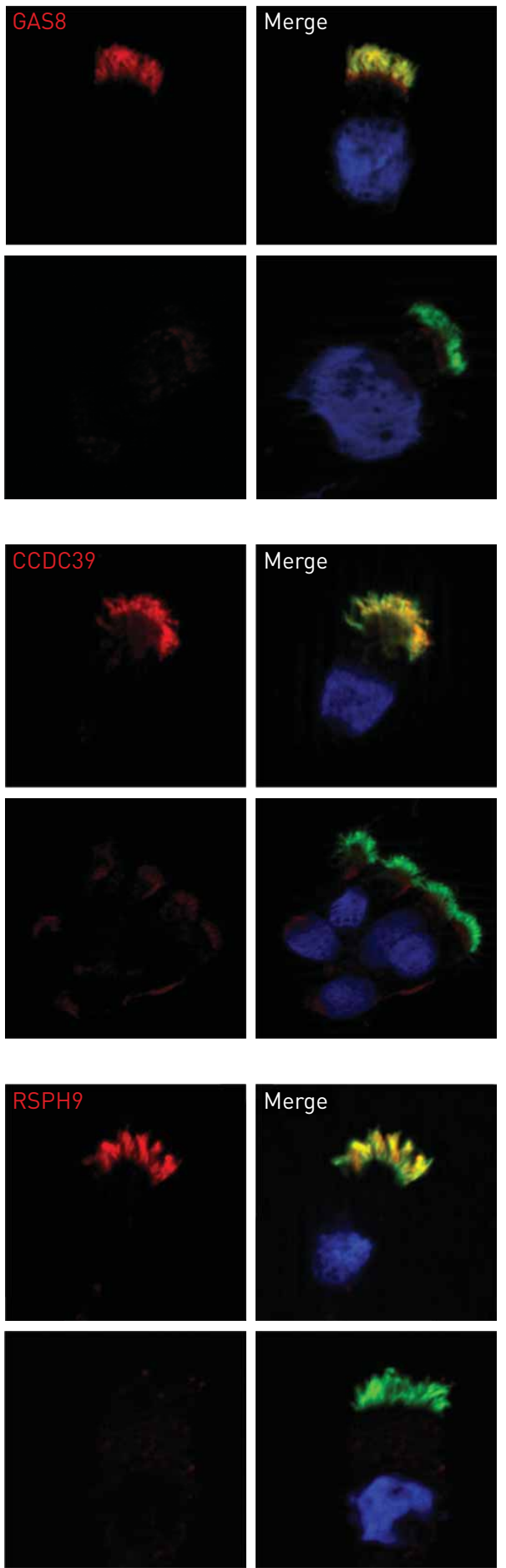
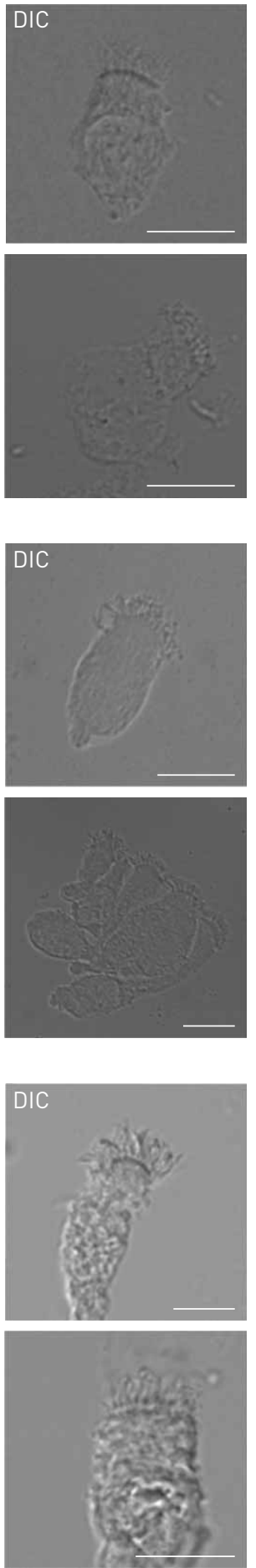

FIGURE 3 Immunofluorescence microscopy can be used to identify structural defects of motile cilia and to aid diagnosis of primary ciliary dyskinesia. Three examples of abnormal staining are presented. a) Antibodies directed against the outer dynein arm heavy chain DNAH5 (green) are used to detect the outer dynein arm complex along the entire ciliary lengths within the control and a person carrying a GAS8 Mutation. The antibodies directed against GAS8 (red) can identify isolated defects of the nexin-dynein regulatory complex. b) Antibodies against CCDC39 (red) can detect defects of the 96-nm axonemal ruler, which consists of CCDC39 and CCDC40. Anti-acetylated tubulin (green) antibodies are used to depict the cilia. c) Antibodies against RSPH9 (red) can be used to identify various defects of the radial spoke head complex. Normal localisation of ciliary components is shown by co-localisation (yellow colour) with ciliary axonemal markers such as acetylated tubulin (green in b and c) or unaffected ciliary components (i.e. DNAH5, green in a). In contrast, absence of structural components involved in ciliary motility is shown by absence of the protein in mutant cells (lower panels in a-c). Nuclei are shown in blue. DIC: differential interference contrast microscopy. Scale bars $=10 \mu \mathrm{m}$. 
Review of evidence directly addressing the question "in patients suspected of having PCD, should immunofluorescence be used as a diagnostic tool?"

Our search identified 276 studies (figure S1). No studies reported use of immunofluorescence antibodies in a diagnostic setting and we were, therefore, unable to establish the accuracy of IF as a diagnostic test. 40 studies contributed to our understanding of the potential to use immunofluorescence diagnostically, as summarised below (table S9).

\section{Narrative review of additional evidence}

Although the literature focuses on research to understand the downstream effects of mutations in PCD-related genes (table 7), several centres now use immunofluorescence to aid diagnosis [117]. This is likely to increase as more antibodies become available, and once data for the accuracy of the tests becomes available. In the two largest patient-based cohort studies, DNAH5 immunofluorescence was tested in 16 PCD patients and a further 17 families who had ODA defects observed by TEM; mislocalisation of the protein was reported in all cases. Furthermore, DNAH5 protein was present in patients with cystic fibrosis and in healthy controls $[94,95]$. A number of studies have used immunofluorescence to examine protein mislocalisation related to genetic mutations (table 7), providing indicators to the antibodies that might be used and findings expected when immunofluorescence is used as a diagnostic test. Immunofluorescence can identify mislocalisation of proteins in PCD patients with a range of mutations, providing information on the pathogenicity of a mutation [86]. However, most manuscripts report immunofluorescence findings from small numbers of patients for each gene and mutation specific findings are not yet known.

With immunofluorescence it is possible to identify almost all ultrastructural abnormalities detectable by TEM and also some cases where the TEM is apparently normal or subtly abnormal $[30,86,97]$. The sensitivity and specificity of immunofluorescence is unknown but will reflect the combination and quality of antibodies. In the authors' experience, a number of antibodies do not work and validation including appropriate disease and healthy controls is required before they are used diagnostically. Immunofluorescence analysis can be normal if the mutated protein is still expressed within the axoneme [97].

We did not find evidence to either confirm or refute immunofluorescence as a diagnostic test for PCD. Whilst further evidence in a diagnostic setting is required, a summary of the Task Force findings from published evidence are shown in table 9.

Key unanswered questions and research needs

We need validation studies to investigate the accuracy and limitations of immunofluorescence as a diagnostic tool for PCD in diagnostic cohort studies. Each applied antibody needs validation in studies including appropriate PCD disease and healthy controls.

\section{Summary}

We were unable to determine the accuracy of immunofluorescence testing due to lack of suitable studies. Task force experts agree that immunofluorescence can be useful in clinical settings. Immunofluorescence is cheaper and easier than other diagnostic tests, providing a potential test for resource-limited settings.

\section{Confirming or excluding a diagnosis of PCD}

The Delphi Consensus Survey comprised four consecutive online surveys, each building on former rounds. The outcomes of each round are summarised in table S11. Experts from the ERS Task Force agreed $(\geqslant 80 \%$ of respondents) on the following, which enabled us to propose a diagnostic algorithm (figure 4).

\section{Positive diagnosis}

For patients with a supportive history of PCD, the following results are confirmatory of a positive diagnosis of PCD.

1) Hallmark ciliary ultrastructure defects for PCD (absence of ODA, combined absence of IDA and ODA, IDA absence combined with microtubular disarrangement) assessed by TEM.

2) Non-ambiguous bi-allelic mutations in PCD causing genes.

The Task Force did not reach consensus $(80 \%)$ that any other test in isolation or in combination could provide a conclusive positive diagnosis.

\section{Highly likely diagnosis}

In patients with a compatible history of PCD, the following diagnostic test results make the diagnosis of PCD highly likely, but do not provide a definitive PCD diagnosis. 
TABLE 9 Summary of the Task Force consensus on the published evidence on immunofluorescence testing in primary ciliary dyskinesia (PCD) diagnostics

Whilst further evidence in a diagnostic setting is required, experts of the Task Force agreed:

1. Immunofluorescence is able to confirm pathogenesis of mutations (e.g. missense mutations in genes encoding radial spoke proteins)

2. Immunofluorescence can detect PCD in some cases with normal ultrastructure or subtle ultrastructural defects

3. Immunofluorescence can help establish the diagnosis of PCD in outer and inner dynein arms, tubular disorganisation (CCDC39/CCDC40 mutations), central pair (genes encoding radial spoke proteins) and nexin link defects

1) Very low nNO plus HSVA findings consistently suggestive of PCD (e.g. static cilia or circling) on three occasions.

2) Very low nNO plus HSVA findings consistent with PCD (e.g. static cilia or circling) following cell culture.

If the diagnosis is "highly likely" but not conclusive, patients should be told that the diagnosis is likely but given the limitations of diagnostic tests, the diagnosis is not $100 \%$ certain and might need confirmation when better tests become available. Patients should have other causes for their symptoms excluded and should be treated as if they have PCD. As new diagnostic tests become available further investigations should be offered.

\section{Excluding the diagnosis of PCD}

The Task Force did not reach consensus (80\%) that any single test or combination of tests could exclude a diagnosis of PCD. However, based on the evidence reviewed they agreed that there are conditions under which the diagnosis is "extremely unlikely". If the clinical suspicion is only modest and nNO is high/ normal plus normal HSVA or nNO is high/normal plus normal HSVA following cell culture.

The patient can be counselled that the diagnosis is extremely unlikely and that further testing is not warranted. If the clinical suspicion is very high (e.g. Kartagener's syndrome, PICADAR score $\geqslant 10$ ) current diagnostic tests are not sufficiently accurate to exclude a diagnosis.

\section{General statements}

Members of the Task Force suggest that diagnostic tests should only be conducted in laboratories with expertise in the field. The results should be interpreted by specialists with expertise in PCD and the results explained to the patient and their non-specialist carers. Diagnostic tests for PCD are currently imperfect. As our understanding and techniques for PCD advance, patients with a high clinical suspicion or inconclusive test results can be recalled and offered repeat testing.

A number of patients have diagnostic tests which do not satisfy the criteria for being labelled positive, highly likely or extremely unlikely. These patients should be considered inconclusive; further investigation and management should be determined by a specialist with expertise in PCD.

\section{Diagnostic algorithm}

Based on the culmination of evidence from the GRADE recommendations and the Delphi Consensus statement, the following step-wise approach to diagnostic testing can be used (figure 4). Not all patients need to undergo all steps. For many patients, step 1 (nNO and HSVA) will provide a "highly unlikely" diagnosis, and patients won't need further investigations. Some patients should proceed to step 2 (TEM or cell culture with repeat HSVA). Genetics testing (step 3) may help make a diagnosis in patients where other tests have failed to provide a definitive diagnostic outcome. Patients who remain "inconclusive" could be recalled in the future as further tests become available. This approach will not be appropriate for all diagnostic services; local expertise and equipment should be taken into consideration.

\section{Step 1: nNO and HSVA}

If both are entirely normal, the diagnosis of PCD is very unlikely and further testing can be avoided unless the clinical suspicion is particularly high. If $\mathrm{nNO}$ is low and/or HSVA is abnormal PCD is the likely/ possible diagnosis. Repeat the step 1 tests and proceed to step 2.

Step 2: TEM

If TEM is normal consider genetics testing for genes associated with normal or subtle TEM defects and repeat HSVA following cell culture. If TEM shows "hallmark" defects PCD is confirmed, consider genetics testing to further characterise the underlying defect. 


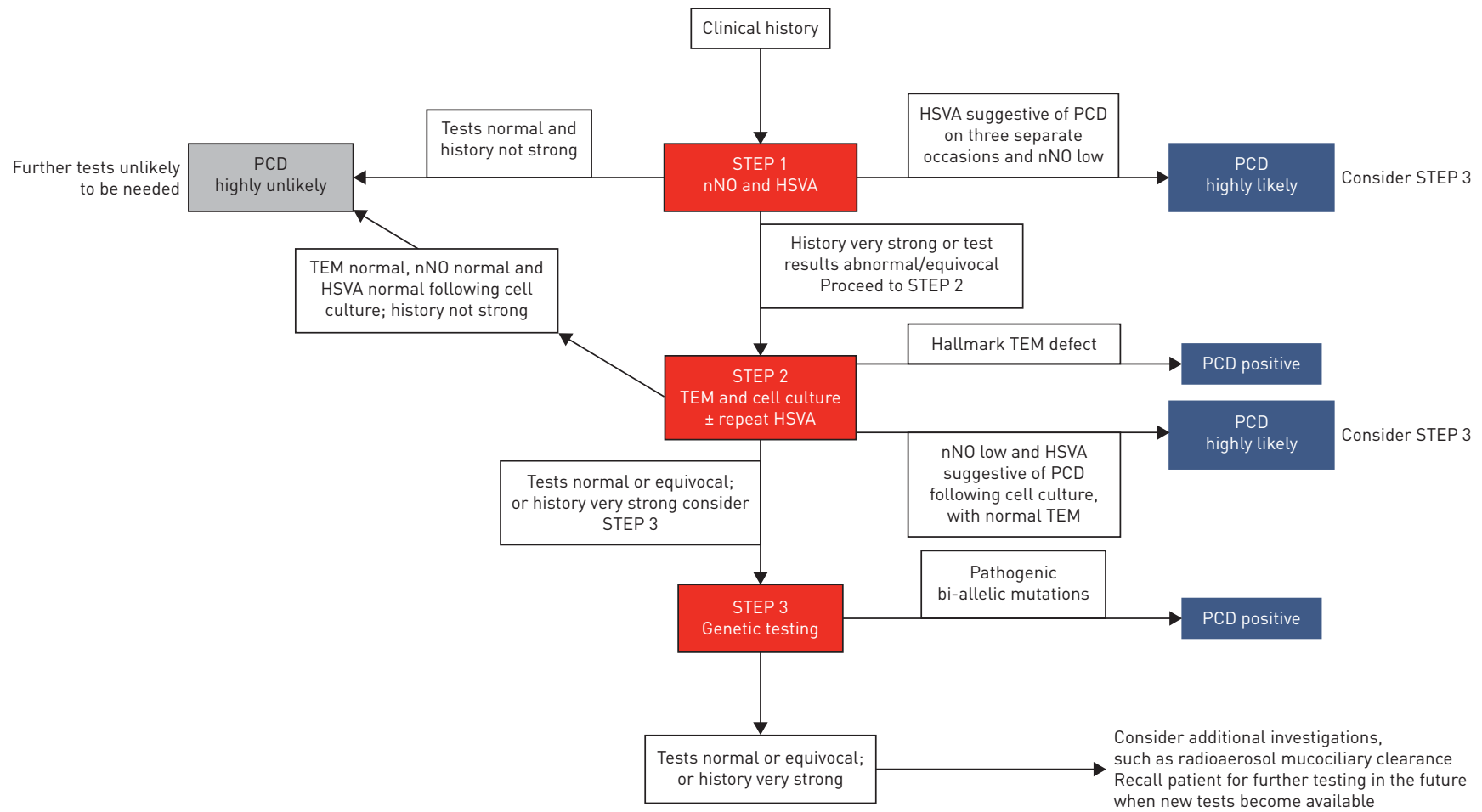

FIGURE 4 Following development of recommendations using the GRADE (Grading of Recommendations, Assessment, Development and Evaluation) approach, a Delphi survey allowed us to propose a diagnostic algorithm for primary ciliary dyskinesia (PCD). Not all patients need to go through all steps. Please see the text for details of the implications of each diagnostic outcome (positive, highly likely and highly unlikely), as well as the consequences for patients who will continue to have an inconclusive outcome using currently available diagnostic tests. Patients with uncertain outcomes should be reconsidered for further testing as advances in diagnostic tests are made. nNO: nasal nitric oxide; TEM: transmission electron microscopy; HSVA: high-speed video microscopy analysis.

Step 3: Genetics and repeat HSVA \pm cell culture.

\section{Further testing}

In patients where the diagnosis is highly likely or remains inconclusive, further investigations such as immunofluorescence or radio-aerosol mucociliary clearance analysis might be used but the evidence is too limited for us to recommend them. PCD clinicians should consider recalling these patients for further testing in the future, as advances in PCD diagnostics are made.

\section{Discussion}

The ERS Task Force presents the first evidence-based guideline for the diagnosis of PCD. This is timely, as new diagnostic tests (e.g. ciliary protein immunofluorescence and genetic testing) are increasingly introduced along with refinement of existing tests (e.g. electron microscopy tomography and computational averaging). As new evidence arises, the guidelines will need revisiting. We have provided guidance on who should be referred for diagnostic testing. We have confirmed that no diagnostic test is perfect, and in the absence of a gold standard, access to a combination of tests is necessary. Using a modified Delphi approach we then used the evidence for individual tests to develop a diagnostic algorithm, providing the criteria to define patients as positive, highly unlikely, extremely unlikely and inconclusive.

The studies that contribute to the recommendations were all conducted in specialist PCD diagnostic centres. The tests are generally complex, requiring experienced scientists and clinicians to analyses and interpret results. Therefore, our findings provide evidence for diagnostic centres with high throughput of samples, analysed by experienced technicians and with good quality control. New diagnostic centres will require support from experienced centres for training and ongoing quality control/assessment. BEATPCD (COST ACTION BM 1407; www.beatpcd.org/) is coordinating a programme of research and training to improve the diagnosis and treatment of PCD. This includes provision of training schools, bursaries for short-term placements in specialist centres and networking for discussion of difficult and equivocal diagnostic decisions. Together with the anticipated European Reference Network for PCD, the collaborative approach should drive up standards of diagnostic testing across Europe. 
Results from diagnostic studies are an indirect measure of the downstream consequences of the application of a test, and therefore the confidence in the test accuracy measures was judged, at best, as moderate to low. Evidence from patients does not alter the strength of published evidence but was considered when deciding the strength of recommendations. The Task Force conducted a questionnaire survey of 352 PCD patients from 25 countries, and 20 in-depth interviews [7]. Patients told us that an accurate diagnosis was an important outcome, leading to a better recognition of their problems by physicians and access to effective treatment, thus improving their health and quality of life.

Once the Task Force recommendations were agreed, a modified Delphi survey was used to develop a consensus on diagnostic approach. Experts from the Task Force agreed that results from TEM and genetic testing can lead to a definite positive diagnosis. This provides a guide for future clinical trials as well as clinical care, with the caution that our definition will systematically exclude PCD patients with normal ciliary ultrastructure where the genetic mutations are not yet known. Furthermore, most of the Task Force $(>50 \%)$ considered that the following combinations of tests could lead to a positive diagnosis, but we did not reach consensus (defined by $\geqslant 80 \%$ ): very low nNO plus hallmark HSVA consistently on two occasions and very low nNO plus hallmark HSVA following cell culture (table S11). We have also defined conditions where the diagnosis of PCD can be considered as "extremely likely" and where diagnosis is "extremely unlikely". Given the current evidence, it is not possible to exclude a diagnosis with $100 \%$ confidence, but we have defined situations where further testing can reasonably stop. We anticipate that as our understanding of PCD grows, new phenotypes for this highly heterogeneous condition will be described that might not be detected by current diagnostic tests.

All studies that contribute to our recommendations were hampered by the lack of a gold standard to investigate the accuracy of individual tests. We therefore accepted manuscripts that constructed a reference standard from a number of tests (composite diagnostic outcome) or used an imperfect test, e.g. TEM as a surrogate [118]. Studies using TEM or genetics as the reference standard will systematically exclude PCD patients with normal ultrastructure or where the genetic mutation is not known. Additionally, some of the evidence was based on studies where the index test was included in the composite diagnostic decision. These important limitations present a strong risk of bias and may have over inflated or deflated the sensitivity and specificity that we report for each test. None of the diagnostic tests had internationally agreed standards for conduct or reporting. This resulted in disparity of methods between the studies that we reviewed. The Task Force suggests that the following need to be taken into consideration in order to advance our understanding of diagnostic tests for PCD.

1) Methodologists should be involved in the design of future studies to investigate diagnostic accuracy of tests. Consideration is needed for the lack of a perfect reference standard.

2) To allow comparisons between studies, international standards for conduct of diagnostic tests and reporting of results is needed. The standards should be evidence based.

3) Reporting of the clinical phenotype of patients included in diagnostic studies should be improved and standardised.

4) Impact of a diagnosis on patient outcome and quality of life should be investigated.

\section{Acknowledgements}

The Task Force members are also participants in BEAT-PCD (COST Action BM 1407).

\section{References}

1 Lucas JS, Leigh MW. Diagnosis of primary ciliary dyskinesia: searching for a gold standard. Eur Respir J 2014; 44: 1418-1422.

2 Barbato A, Frischer T, Kuehni CE, et al. Primary ciliary dyskinesia: a consensus statement on diagnostic and treatment approaches in children. Eur Respir J 2009; 34: 1264-1276.

3 Kuehni CE, Frischer T, Strippoli MP, et al. Factors influencing age at diagnosis of primary ciliary dyskinesia in European children. Eur Respir J 2010; 36: 1248-1258.

4 Strippoli MP, Frischer T, Barbato A, et al. Management of primary ciliary dyskinesia in European children: recommendations and clinical practice. Eur Respir J 2012; 39: 1482-1491.

5 Schünemann HJ, Oxman AD, Brozek J, et al. Grading quality of evidence and strength of recommendations for diagnostic tests and strategies. BMJ 2008; 336: 1106-1110.

6 Guyatt GH, Oxman AD, Kunz R, et al. GRADE guidelines: 2. Framing the question and deciding on important outcomes. J Clin Epidemiol 2011; 64: 395-400.

7 Behan L, Dunn Galvin A, Rubbo B, et al. Diagnosing primary ciliary dyskinesia; an international patient perspective. Eur Respir J 2016; 48: 1096-1107.

8 Balshem H, Helfand M, Schünemann HJ, et al. GRADE guidelines: 3. Rating the quality of evidence. J Clin Epidemiol 2011; 64: 401-406.

9 Andrews JC, Schünemann HJ, Oxman AD, et al. GRADE guidelines: 15. Going from evidence to recommendation: determinants of a recommendation's direction and strength. J Clin Epidemiol 2013; 66: 726-735. 

Eur Respir I 2016; 47: 1103-1112. Shapiro AJ, Chawla KK, Baker BR, et al. Nasal nitric oxide and clinical characteristics of patients with heterotaxy: comparison to primary ciliary dyskinesia. Am J Respir Crit Care Med 2011; 183: A1209.

12 Leigh MW, Ferkol TW, Davis SD, et al. Clinical features and associated likelihood of primary ciliary dyskinesia in children and adolescents. Ann Am Thorac Soc 2016; 13: 1305-1313.

13 Noll EM, Rieger CHL, Hamelmann E, et al. Questionnaire to preselect patients with a high probability of primary ciliary dyskinesia. Klin Pädiatrie 2011; 223: 22-26.

14 Chin GY, Karas DE, Kashgarian M. Correlation of presentation and pathologic condition in primary ciliary dyskinesia. Arch Otolaryngol Head Neck 02; 128: 1292-1294.

15 Beucher J, Chambellan A, Segalen J, et al. Primary ciliary dyskinesia: a retrospective review of clinical and paraclinical data. Rev Mal Respir 2011; 28: 856-863.

16 Pifferi M, Bush A, Michelucci A, et al. Mannose-binding lectin 2 gene polymorphism and lung damage in primary ciliary dyskinesia. Pediatr Pulmonol 2015; 50: 179-186.

17 Mullowney T, Manson D, Kim R, et al. Primary ciliary dyskinesia and neonatal respiratory distress. Pediatrics 2014; 134: 1160-1166.

18 Goutaki M, Meier AB, Halbeisen FS, et al. Clinical manifestations in primary ciliary dyskinesia: a systematic review and meta-analysis. Eur Respir J 2016; 48: 1081-1095.

19 Walker WT, Jackson CL, Lackie PM, et al. Nitric oxide in primary ciliary dyskinesia. Eur Respir J 2012; 40: 1024-1032.

20 Collins SA, Gove K, Walker W, et al. Nasal nitric oxide screening for primary ciliary dyskinesia: systematic review and meta-analysis. Eur Respir J 2014; 44: 1589-1599.

21 American Thoracic Society, European Respiratory Society. ATS/ERS recommendations for standardized procedures for the online and offline measurement of exhaled lower respiratory nitric oxide and nasal nitric oxide, 2005. Am J Respir Crit Care Med 2005; 171: 912-930.

22 Beydon N, Chambellan A, Alberti C, et al. Technical and practical issues for tidal breathing measurements of nasal nitric oxide in children. Pediatr Pulmonol 2015; 50: 1374-1382.

23 Marthin JK, Nielsen KG. Hand-held tidal breathing nasal nitric oxide measurement - a promising targeted case-finding tool for the diagnosis of primary ciliary dyskinesia. PLoS One 2013; 8: e57262.

24 Marthin JK, Nielsen KG. Choice of nasal nitric oxide technique as first-line test for primary ciliary dyskinesia. Eur Respir J 2011; 37: 559-565.

25 Leigh MW, Hazucha MJ, Chawla KK, et al. Standardizing nasal nitric oxide measurement as a test for primary ciliary dyskinesia. Ann Am Thorac Soc 2013; 10: 574-581.

26 Jackson CL, Behan L, Collins SA, et al. Accuracy of diagnostic testing in primary ciliary dyskinesia. Eur Respir J 2016; 47: 837-848

27 Harris A, Bhullar E, Gove K, et al. Validation of a portable nitric oxide analyzer for screening in primary ciliary dyskinesias. BMC Pulm Med 2014; 14: 18

28 Struben VM, Wieringa MH, Mantingh CJ, et al. Nasal NO: normal values in children age 6 through to 17 years. Eur Respir J 2005; 26: 453-457.

29 Adams PS, Tian X, Zahid M, et al. Establishing normative nasal nitric oxide values in infants. Respir Med 2015 109: 1126-1130.

30 Olbrich H, Cremers C, Loges NT, et al. Loss-of-function GAS8 mutations cause primary ciliary dyskinesia and disrupt the nexin-dynein regulatory complex. Am J Hum Genet 2015; 97: 546-554.

31 Knowles MR, Ostrowski LE, Leigh MW, et al. Mutations in RSPH1 cause primary ciliary dyskinesia with a unique clinical and ciliary phenotype. Am J Respir Crit Care Med 2014; 189: 707-717.

32 Kott E, Legendre M, Copin B, et al. Loss-of-function mutations in RSPH1 cause primary ciliary dyskinesia with central-complex and radial-spoke defects. Am J Hum Genet 2013; 93: 561-570.

33 Afzelius BA. A human syndrome caused by immotile cilia. Science 1976; 193: 317-319.

34 Rayner CF, Rutman A, Dewar A, et al. Ciliary disorientation alone as a cause of primary ciliary dyskinesia syndrome. Am J Respir Crit Care Med 1996; 153: 1123-1129.

35 Chapelin C, Coste A, Reinert P, et al. Incidence of primary ciliary dyskinesia in children with recurrent respiratory diseases. Ann Otol Rhinol Laryngol 1997; 106: 854-858.

36 Santamaria MM, de Santi G, Grillo F. Ciliary motility at light microscopy: a screening technique for ciliary defects? Acta Paediatr 1999; 88: 853-857.

37 Jorissen M, Willems T, Van der Schueren B, et al. Ultrastructural expression of primary ciliary dyskinesia after ciliogenesis in culture. Acta Otorhinolaryngol Belg 2000; 54: 343-356.

38 Toskala-Hannikainen E, Haataja J, Shirasaki H, et al. Culture of cells harvested with nasal brushing: a method for evaluating ciliary function. Rhinology 2005; 43: 121-124.

39 Pifferi M, Montemurro F, Cangiotti AM, et al. Simplified cell culture method for the diagnosis of atypical primary ciliary dyskinesia. Thorax 2009; 64: 1077-1081.

40 Hirst RA, Jackson CL, Coles JL, et al. Culture of primary ciliary dyskinesia epithelial cells at air-liquid interface can alter ciliary phenotype but remains a robust and informative diagnostic aid. PLoS One 2014; 9: e89675.

41 Chilvers MA, O'Callaghan C. Analysis of ciliary beat pattern and beat frequency using digital high speed imaging: comparison with the photomultiplier and photodiode methods. Thorax 2000; 55: 314-317.

42 Papon J-F, Bassinet L, Cariou-Patron G, et al. Quantitative analysis of ciliary beating in primary ciliary dyskinesia: a pilot study. Orphanet J Rare Dis 2012; 7: 78

43 Friedman NR, Pachigolla R, Deskin RW, et al. Optimal technique to diagnose primary ciliary dyskinesia. Laryngoscope 2000; 110: 1548-1551.

44 Chilvers MA, Rutman A, O'Callaghan C. Ciliary beat pattern is associated with specific ultrastructural defects in primary ciliary dyskinesia. J Allergy Clin Immunol 2003; 112: 518-524.

45 Raidt J, Wallmeier J, Hjeij R, et al. Ciliary beat pattern and frequency in genetic variants of primary ciliary dyskinesia. Eur Respir J 2014; 44: 1579-1588.

46 Pifferi M, Bush A, Montemurro F, et al. Rapid diagnosis of primary ciliary dyskinesia: cell culture and soft computing analysis. Eur Respir J 2013; 41: 960-965. 
Jorissen M, Willems T, Van der Schueren B. Ciliary function analysis for the diagnosis of primary ciliary dyskinesia: advantages of ciliogenesis in culture. Acta Otolaryngol 2000; 120: 291-295.

Stannard WA, Chilvers MA, Rutman AR, et al. Diagnostic testing of patients suspected of primary ciliary dyskinesia. Am J Respir Crit Care Med 2010; 181: 307-314.

Schwabe GC, Hoffmann K, Loges NT, et al. Primary ciliary dyskinesia associated with normal axoneme ultrastructure is caused by DNAH11 mutations. Hum Mutat 2008; 29: 289-298.

Sturgess JM, Turner JA. Ultrastructural pathology of cilia in the immotile cilia syndrome. Perspect Pediatr Pathol 1984; 8: 133-161.

Rutland J, Dewar A, Cox T, et al. Nasal brushing for the study of ciliary ultrastructure. J Clin Pathol 1982; 35 357-359.

Pifferi M, Caramella D, Cangiotti AM, et al. Nasal nitric oxide in atypical primary ciliary dyskinesia. Chest 2007 131: $870-873$

Hirst RA, Rutman A, Williams G, et al. Ciliated air-liquid cultures as an aid to diagnostic testing of primary ciliary dyskinesia. Chest 2010; 138: 1441-1447.

Papon JF, Coste A, Roudot-Thoraval F, et al. A 20-year experience of electron microscopy in the diagnosis of primary ciliary dyskinesia. Eur Respir J 2010; 35: 1057-1063.

Olm MA, Kögler JE, Macchione M, et al. Primary ciliary dyskinesia: evaluation using cilia beat frequency assessment via spectral analysis of digital microscopy images. J Appl Physiol 2011; 111: 295-302.

Shoemark A, Dixon M, Corrin B, et al. Twenty-year review of quantitative transmission electron microscopy for the diagnosis of primary ciliary dyskinesia. J Clin Pathol 2012; 65: 267-271.

Munkholm M, Nielsen KG, Mortensen J. Clinical value of measurement of pulmonary radioaerosol mucociliary clearance in the work up of primary ciliary dyskinesia. EJNMMI Res 2015; 5: 118.

Olin JT, Burns K, Carson JL, et al. Diagnostic yield of nasal scrape biopsies in primary ciliary dyskinesia: a multicenter experience. Pediatr Pulmonol 2011; 46: 483-488.

Boon M, Smits A, Cuppens H, et al. Primary ciliary dyskinesia: critical evaluation of clinical symptoms and diagnosis in patients with normal and abnormal ultrastructure. Orphanet J Rare Dis 2014; 9: 11.

Escudier E, Couprie M, Duriez B, et al. Computer-assisted analysis helps detect inner dynein arm abnormalities. Am J Respir Crit Care Med 2002; 166: 1257-1262.

Funkhouser WK, Niethammer M, Carson JL, et al. A new tool improves diagnostic test performance for transmission em evaluation of axonemal dynein arms. Ultrastruct Pathol 2014; 38: 248-255.

Smith CM, Hirst RA, Bankart MJ, et al. Cooling of cilia allows functional analysis of the beat pattern for diagnostic testing. Chest 2011; 140: 186-190.

Shoemark A, Hogg C. Electron tomography of respiratory cilia. Thorax 2013; 68: 190-191.

Wallmeier J, Al-Mutairi DA, Chen C-T, et al. Mutations in CCNO result in congenital mucociliary clearance disorder with reduced generation of multiple motile cilia. Nat Genet 2014; 46: 646-651.

Olbrich H, Häffner K, Kispert A, et al. Mutations in DNAH5 cause primary ciliary dyskinesia and randomization of left-right asymmetry. Nat Genet 2002; 30: 143-144.

Pennarun G, Escudier E, Chapelin C, et al. Loss-of-function mutations in a human gene related to Chlamydomonas reinhardtii dynein IC78 result in primary ciliary dyskinesia. Am J Hum Genet 1999; 65: 1508-1519.

Loges NT, Olbrich H, Fenske L, et al. DNAI2 mutations cause primary ciliary dyskinesia with defects in the outer dynein arm. Am J Hum Genet 2008; 83: 547-558.

Duriez B, Duquesnoy P, Escudier E, et al. A common variant in combination with a nonsense mutation in member of the thioredoxin family causes primary ciliary dyskinesia. Proc Natl Acad Sci USA 2007; 104: 3336-3341.

Mazor M, Alkrinawi S, Chalifa-Caspi V, et al. Primary ciliary dyskinesia caused by homozygous mutation in DNAL1, encoding dynein light chain 1. Am J Hum Genet 2011; 88: 599-607.

Hjeij R, Onoufriadis A, Watson CM, et al. CCDC151 mutations cause primary ciliary dyskinesia by disruption of the outer dynein arm docking complex formation. Am J Hum Genet 2014; 95: 257-274.

Onoufriadis A, Paff T, Antony D, et al. Splice-site mutations in the axonemal outer dynein arm docking complex gene CCDC114 cause primary ciliary dyskinesia. Am J Hum Genet 2013; 92: 88-98.

(1) Shoemark A, Munye MM, et al. Combined exome and whole-genome sequencing identifies mutations in ARMC4 as a cause of primary ciliary dyskinesia with defects in the outer dynein arm. J Med Genet 2014; 51: 61-67. Panizzi JR, Becker-Heck A, Castleman VH, et al. CCDC103 mutations cause primary ciliary dyskinesia by disrupting assembly of ciliary dynein arms. Nat Genet 2012; 44: 714-719.

Tarkar A, Loges NT, Slagle CE, et al. DYX1C1 is required for axonemal dynein assembly and ciliary motility. Nat Genet 2013; 45: 995-1003.

Knowles MR, Ostrowski LE, Loges NT, et al. Mutations in SPAG1 cause primary ciliary dyskinesia associated with defective outer and inner dynein arms. Am J Hum Genet 2013; 93: 711-720.

Horani A, Ferkol TW, Shoseyov D, et al. LRRC6 mutation causes primary ciliary dyskinesia with dynein arm defects. PLoS One 2013; 8: e59436.

Omran $\mathrm{H}$, Kobayashi $\mathrm{D}$, Olbrich $\mathrm{H}$, et al. Ktu/PF13 is required for cytoplasmic pre-assembly of axonema dyneins. Nature 2008; 456: 611-616.

Duquesnoy P, Escudier E, Vincensini L, et al. Loss-of-function mutations in the human ortholog of Chlamydomonas reinhardtii ODA7 disrupt dynein arm assembly and cause primary ciliary dyskinesia. Am J Hum Genet 2009; 85: 890-896.

Loges NT, Olbrich H, Becker-Heck A, et al. Deletions and point mutations of LRRC50 cause primary ciliary dyskinesia due to dynein arm defects. Am J Hum Genet 2009; 85: 883-889.

Austin-Tse C, Halbritter J, Zariwala MA, et al. Zebrafish ciliopathy screen plus human mutational analysis identifies C21orf59 and CCDC65 defects as causing primary ciliary dyskinesia. Am J Hum Genet 2013; 93: 672-686.

Mitchison HM, Schmidts M, Loges NT, et al. Mutations in axonemal dynein assembly factor DNAAF3 cause primary ciliary dyskinesia. Nat Genet 2012; 44: 381-389.

Zariwala MA, Gee HY, Kurkowiak M, et al. ZMYND10 is mutated in primary ciliary dyskinesia and interacts with LRRC6. Am J Hum Genet 2013; 93: 336-345

Horani A, Druley TE, Zariwala MA, et al. Whole-exome capture and sequencing identifies HEATR2 mutation as a cause of primary ciliary dyskinesia. Am J Hum Genet 2012; 91: 685-693. 
Olbrich H, Schmidts M, Werner C, et al. Recessive HYDIN mutations cause primary ciliary dyskinesia without randomization of left-right body asymmetry. Am J Hum Genet 2012; 91: 672-684.

Jeanson L, Copin B, Papon J-F, et al. RSPH3 mutations cause primary ciliary dyskinesia with central-complex defects and a near absence of radial spokes. Am J Hum Genet 2015; 97: 153-162.

Frommer A, Hjeij R, Loges NT, et al. Immunofluorescence analysis and diagnosis of primary ciliary dyskinesia with radial spoke defects. Am J Respir Cell Mol Biol 2015; 53: 563-573.

Wirschell M, Olbrich $\mathrm{H}$, Werner $\mathrm{C}$, et al. The nexin-dynein regulatory complex subunit DRC1 is essential for motile cilia function in algae and humans. Nat Genet 2013; 45: 262-268.

Horani A, Brody SL, Ferkol TW, et al. CCDC65 mutation causes primary ciliary dyskinesia with normal ultrastructure and hyperkinetic cilia. PLoS One 2013; 8: e72299.

Merveille AC, Davis EE, Becker-Heck A, et al. CCDC39 is required for assembly of inner dynein arms and the dynein regulatory complex and for normal ciliary motility in humans and dogs. Nat Genet 2011; 43: 72-78.

Becker-Heck A, Zohn IE, Okabe N, et al. The coiled-coil domain containing protein CCDC40 is essential for motile cilia function and left-right axis formation. Nat Genet 2011; 43: 79-84.

Moore A, Escudier E, Roger G, et al. RPGR is mutated in patients with a complex X linked phenotype combining primary ciliary dyskinesia and retinitis pigmentosa. J Med Genet 2006; 43: 326-333.

Budny B, Chen W, Omran $\mathrm{H}$, et al. A novel X-linked recessive mental retardation syndrome comprising macrocephaly and ciliary dysfunction is allelic to oral-facial-digital type I syndrome. Hum Genet 2006; 120: 171-178.

Boon M, Wallmeier J, Ma L, et al. MCIDAS mutations result in a mucociliary clearance disorder with reduced generation of multiple motile cilia. Nat Commun 2014; 5: 4418.

Fliegauf M, Olbrich $\mathrm{H}$, Horvath J, et al. Mislocalization of DNAH5 and DNAH9 in respiratory cells from patients with primary ciliary dyskinesia. Am J Respir Crit Care Med 2005; 171: 1343-1349.

Hornef $\mathrm{N}$, Olbrich $\mathrm{H}$, Horvath J, et al. DNAH5 mutations are a common cause of primary ciliary dyskinesia with outer dynein arm defects. Am J Respir Crit Care Med 2006; 174: 120-126.

Knowles MR, Leigh MW, Carson JL, et al. Mutations of DNAH11 in patients with primary ciliary dyskinesia with normal ciliary ultrastructure. Thorax 2012; 67: 433-441.

Dougherty GW, Loges NT, Klinkenbusch JA, et al. DNAH11 localization in the proximal region of respiratory cilia defines distinct outer dynein arm complexes. Am J Respir Cell Mol Biol 2016; 55: 213-224.

Hjeij R, Lindstrand A, Francis R, et al. ARMC4 mutations cause primary ciliary dyskinesia with randomization of left/right body asymmetry. Am J Hum Genet 2013; 93: 357-367.

Kott E, Duquesnoy P, Copin B, et al. Loss-of-function mutations in LRRC6, a gene essential for proper axonemal assembly of inner and outer dynein arms, cause primary ciliary dyskinesia. Am J Hum Genet 2012; 91: 958-964.

Kurkowiak M, Ziętkiewicz E, Greber A, et al. ZMYND10 - mutation analysis in slavic patients with primary ciliary dyskinesia. PLoS One 2016; 11: e0148067.

Diggle CP, Moore DJ, Mali G, et al. HEATR2 plays a conserved role in assembly of the ciliary motile apparatus. PLoS Genet 2014; 10: e1004577.

Onoufriadis A, Shoemark A, Schmidts M, et al. Targeted NGS gene panel identifies mutations in RSPH1 causing primary ciliary dyskinesia and a common mechanism for ciliary central pair agenesis due to radial spoke defects. Hum Mol Genet 2014; 23: 3362-3374.

Antony D, Becker-Heck A, Zariwala MA, et al. Mutations in CCDC39 and CCDC40 are the major cause of primary ciliary dyskinesia with axonemal disorganization and absent inner dynein arms. Hum Mutat 2013; 34: $462-472$.

Zietkiewicz E, Loges NT, Wittmer M, et al. RPGR mutations might cause reduced orientation of respiratory cilia. Pediatr Pulmonol 2013; 48: 352-363.

Zariwala MA, Leigh MW, Ceppa F, et al. Mutations of DNAI1 in primary ciliary dyskinesia: evidence of founder effect in a common mutation. Am J Respir Crit Care Med 2006; 174: 858-866.

Amirav I, Wallmeier J, Loges NT, et al. Systematic analysis of CCNO variants in a defined population: implications for clinical phenotype and differential diagnosis. Hum Mutat 2016; 37: 396-405.

Richards S, Aziz N, Bale S, et al. Standards and guidelines for the interpretation of sequence variants: a joint consensus recommendation of the American College of Medical Genetics and Genomics and the Association for Molecular Pathology. Genet Med 2015; 17: 405-424.

Lucas JS, Adam EC, Goggin PM, et al. Static respiratory cilia associated with mutations in Dnahc11/DNAH11: a mouse model of PCD. Hum Mutat 2012; 33: 495-503.

Davis SD, Ferkol TW, Rosenfeld M, et al. Clinical features of childhood primary ciliary dyskinesia by genotype and ultrastructural phenotype. Am J Respir Crit Care Med 2015; 191: 316-324.

Marshall CR, Scherer SW, Zariwala MA, et al. Whole-exome
primary ciliary dyskinesia. G3 (Bethesda) 2015; 5: 1775-1781.

Djakow J, Svobodová T, Hrach K, et al. Effectiveness of sequencing selected exons of DNAH5 and DNAI1 in diagnosis of primary ciliary dyskinesia. Pediatr Pulmonol 2012; 47: 864-875.

2 Failly M, Bartoloni L, Letourneau A, et al. Mutations in DNAH5 account for only $15 \%$ of a non-preselected cohort of patients with primary ciliary dyskinesia. J Med Genet 2009; 46: 281-286.

Blanchon S, Legendre M, Copin B, et al. Delineation of CCDC39/CCDC40 mutation spectrum and associated phenotypes in primary ciliary dyskinesia. J Med Genet 2012; 49: 410-416.

Claustres M, Kožich V, Dequeker E, et al. Recommendations for reporting results of diagnostic genetic testing (biochemical, cytogenetic and molecular genetic). Eur J Hum Genet 2014; 22: 160-170.

Matthijs G, Souche E, Alders M, et al. Guidelines for diagnostic next-generation sequencing. Eur J Hum Genet 2016; $24: 2-5$.

Omran H, Loges NT. Immunofluorescence staining of ciliated respiratory epithelial cells. Methods Cell Biol 2009; 91: 123-133.

Werner C, Lablans M, Ataian M, et al. An international registry for primary ciliary dyskinesia. Eur Respir J 2016; 47: 849-859.

A A review of methods. Health Technol Assess 2007; 11: iii. 